\title{
Detecting State Changes of Indoor Everyday Objects using Wi-Fi Channel State Information
}

\author{
KAZUYA OHARA, TAKUYA MAEKAWA, and YASUYUKI MATSUSHITA, Osaka University
}

\begin{abstract}
Detecting the events of indoor everyday objects such as door or window open/close events has been actively studied to implement such applications as intrusion detection, adaptive HVAC control, and monitoring an independently living elderly person. This study proposes a method for detecting the events and states of indoor everyday objects such as doors and windows without using distributed sensors attached to the objects. In this study, we achieve practical and unobtrusive event detection using a commodity Wi-Fi access point and a computer equipped with a commodity Wi-Fi module. Specifically, we detect the events using Wi-Fi channel state information (CSI), which describes how a signal propagates from a transmitter to a receiver, and is affected by such events. To handle CSI data that consists of the mixed effects of multiple indoor objects in an environment of interest, we employ independent component analysis to separate the events caused by the objects. The decomposed data are then fed into our event classifier based on convolutional and recurrent neural networks to automatically extract features from CSI data, as it is difficult to intuitively design features to be extracted from the CSI data. Moreover, we correct the neural network estimates by incorporating knowledge about the state transitions of an object using hidden Markov models. For example, because the "open" event of a door occurs only when the door is in a "closed" state. We correct impossible state transitions estimated by the neural network based on this knowledge.
\end{abstract}

CCS Concepts: $\bullet$ Human-centered computing $\rightarrow$ Ubiquitous computing; $\bullet$ Mathematics of computing $\rightarrow$ Kalman filters and hidden Markov models; •Computing methodologies $\rightarrow$ Supervised learning by classification; $\bullet$ Computer systems organization $\rightarrow$ Neural networks;

General Terms: Design, Algorithms, Performance

Additional Key Words and Phrases: Indoor context recognition, open/close event, Wi-Fi channel state information, pattern recognition, deep neural network

ACM Reference format:

Kazuya Ohara, Takuya Maekawa, and Yasuyuki Matsushita. 2017. Detecting State Changes of Indoor Everyday Objects using Wi-Fi Channel State Information. PACM Interact. Mob. Wearable Ubiquitous Technol. 1, 3, Article 88 (September 2017), 28 pages.

DOI: http://doi.org/10.1145/3131898

\section{INTRODUCTION}

Indoor context recognition is one of the most important technologies for context-aware systems such as home automation and elder care support. In the ubicomp research community, distributed ubiquitous sensors have usually been used to sense indoor events and phenomena including the use of objects, temperature, and illumination.

This work is partially supported by JST CREST JPMJCR15E2, JSPS KAKENHI Grant Number JP16H06539, JP26730047, JP17J06602, and JP17H04679.

ACM acknowledges that this contribution was authored or co-authored by an employee, or contractor of the national government. As such, the Government retains a nonexclusive, royalty-free right to publish or reproduce this article, or to allow others to do so, for Government purposes only. Permission to make digital or hard copies for personal or classroom use is granted. Copies must bear this notice and the full citation on the first page. Copyrights for components of this work owned by others than ACM must be honored. To copy otherwise, distribute, republish, or post, requires prior specific permission and/or a fee. Request permissions from permissions@acm.org.

(C) 217 ACM. 2474-9567/2017/9-ART88 $\$ 15.00$

DOI: http://doi.org/10.1145/3131898

Proceedings of the ACM on Interactive, Mobile, Wearable and Ubiquitous Technologies, Vol. 1, No. 3, Article 88. Publication date: September 2017. 


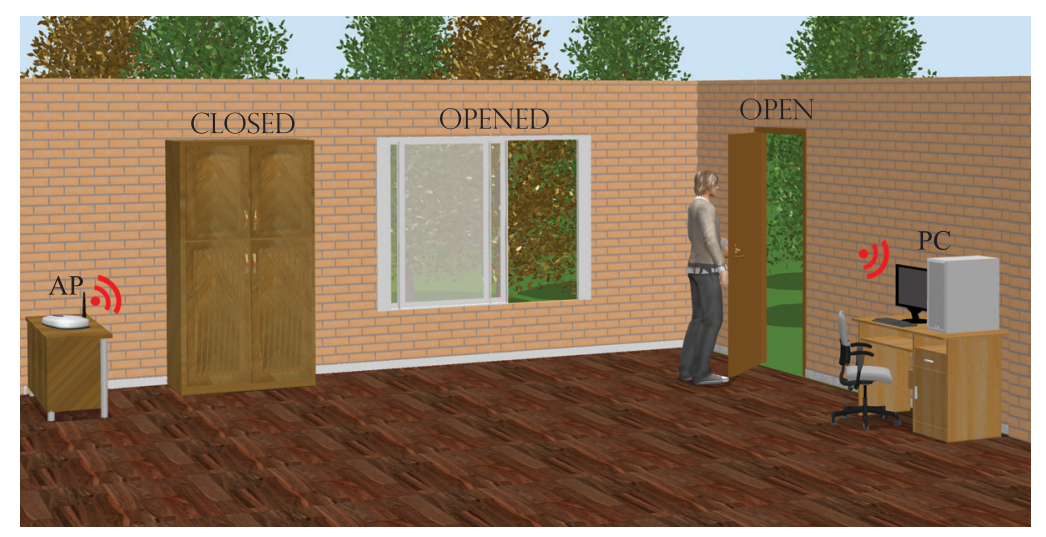

Fig. 1. Detecting events and states of indoor everyday objects using Wi-Fi signals

In particular, sensor systems for detecting the events of indoor everyday objects, e.g., door open/close and window open/close events have been actively studied to implement such applications as intrusion detection, adaptive HVAC control, and monitoring an independently living elderly person. To detect the events and states of such everyday objects, acceleration sensors, gyroscopes, vibration sensors, and state-change sensors (using, e.g., reed switches and magnets) in wireless sensor nodes have been used [32, 40, 41]. However, this distributed sensing approach is expensive to deploy because we must attach a wireless sensor node to each indoor object and maintain these sensor nodes, e.g., replacing node batteries and faulty sensor nodes. When the number of sensor nodes installed in a house is large, the residents could frequently need a maintenance personnel to visit the home and fix faulty nodes. Kodeswaran et al. [24] revealed that houses with 14-100 sensor nodes require a maintenance visit every 18 days on average based on public datasets of IoT enabled homes. In addition, sensor nodes attached to indoor objects can detract from the aesthetics of artifacts in the home [5]. Therefore, event recognition techniques that work well with small numbers of devices are required.

This study proposes a method for detecting events and states of indoor everyday objects such as doors and windows without using distributed sensors attached to the objects. In this study, we achieve practical and unobtrusive event detection using a commodity Wi-Fi access point (AP) and a computer or sensor node equipped with a commodity Wi-Fi module, i.e., a Wi-Fi signal transmitter and receiver pair, in a room. (See Fig. 1.) Specifically, we detect the events using Wi-Fi channel state information (CSI), which describes how a signal propagates from a transmitter to a receiver and is affected by such events.

The currently widely used $802.11 \mathrm{a} / \mathrm{g} / \mathrm{n}$ networks employ orthogonal frequency division multiplexing (OFDM), in which modulated data are transmitted and received in parallel using multiple subcarriers in different frequencies. In addition, data are transmitted and received using multiple antennas based on a multiple input/multiple output (MIMO) processing. The data that travel through the MIMO channel from the transmitter to the receiver are affected by scattering, fading, Doppler shifts, and power decay with distance while traveling, and the channel quality between the transmitter and receiver, which is extracted from the PHY layer in the seven-layer OSI model, is referred to as CSI. CSI is a fine-grained value that describes complex channel information including magnitude and phase information for each subcarrier as well as for each transmit and received antenna in MIMO. Because multiple subcarriers attenuate differently when transmitted through various transmission paths, the amplitude, and phase of each subcarrier represent the physical conditions and events on its transmission path. Nowadays, because it is possible to obtain CSI from some advanced Wi-Fi network interface cards (NIC) such as the Intel $5300 \mathrm{Wi}-\mathrm{Fi}$ 
NIC, CSI has been used in context recognition studies to achieve fine-grained indoor positioning and device-free activity recognition $[10,42,47]$.

Each MIMO channel between each transmit-receive antenna pair of a transmitter and receiver consists of multiple subcarriers. CSI values provided by a NIC characterize the channel impulse response for each subcarrier between each transmit-receive antenna pair. That is, when $M_{T}$ is the number of transmit antennas and $M_{R}$ is the number of receiver antennas, an $M_{T} \times M_{R}$ dimensional channel matrix is provided for each subcarrier. When we have $N_{S}$ subcarriers, $M_{T} \times M_{R} \times N_{S}$ dimensional CSI data are observed for each packet, where each dimension corresponds to CSI for each subcarrier between each transmit-receive antenna pair. Because many existing CSI-based context recognition methods, e.g., indoor positioning and activity recognition, rely on classic machine learning and pattern recognition techniques, these methods first preprocess the high-dimensional time-series data and then extract several features designed by researchers.

For example, Ali et al. [4] apply principal component analysis (PCA) on $N_{S}$-dimensional data for each MIMO channel $\left(N_{S}=30\right)$ to reduce the dimensionality and remove noise. In addition, Chapre et al. [10] sum $M_{T} \times M_{R}$ CSI values for each subcarrier and extract the phase and amplitude information from the sum. Wang et al. [45] aggregate $N_{S}$-dimensional data for each MIMO channel into one single value using their average. Furthermore, Wu et al. [47] aggregate $M_{T} \times M_{R} \times N_{S}$ CSI data into one single value that reflects the estimated distance between a transmitter and receiver $\left(M_{T}=M_{R}=1\right.$ in their system) for fingerprint-based indoor positioning.

However, because CSI describes the combined effect of several physical phenomena such as scattering, fading, Doppler shift, and the power decay of the transmitted signal, it is difficult to intuitively design the features to be extracted from the CSI data and verify their validity. Therefore, we apply feature learning approaches based on deep neural networks (DNNs) to this task. Specifically, we design a novel DNN architecture consisting of convolutional layers and long short-term memory (LSTM) layers that can effectively handle the CSI data. Because the CSIs of several different channels and subcarriers are reportedly correlated with each other when a physical event occurs [4], we employ the convolutional layers to learn meaningful features by convolving the CSIs. In addition, we employ the LSTM layers to learn the temporal dynamics of the event.

Furthermore, unlike many existing CSI-based context recognition studies, we attempt to recognize the events of multiple indoor objects. To handle CSI data consisting of the mixed effects of multiple indoor objects, we employ independent component analysis (ICA) [14] to separate the events caused by the objects. Instead of using raw CSI time-series data as input to the DNN-based classifier, we use ICA to decompose $M_{T} \times M_{R} \times N_{S}$ dimensional time-series data into independent signals from several sources, one of which is an everyday object of interest. The decomposed time-series data are fed into the neural network. A typical application of ICA is the cocktail party problem, where speech signals emitted by speakers are separated from recorded audio data consisting of people talking simultaneously in a room. We can say that the CSI values are mixed signals, in which the outputs from separate independent sources (the effects of daily objects in our case) are mixed, because Wi-Fi packets travel through various transmission paths in the entire room. In ICA, an unmixing matrix that decomposes the mixed signals into independent sources is computed. In our method, we compute an unmixing matrix tailored to each object of interest in the room to detect minute signal changes caused by the object.

Moreover, to further improve the event recognition accuracy, we harness a grammar that describes knowledge about the state transitions of an object. With a door, for example, we can specify that an "open" event occurs only when the door is in a "closed" state. In addition, after the "open" event, the door must transit to an "opened" state. In our proposed method, we decode time-series of DNN predictions using hidden Markov models (HMMs) and incorporate this handcrafted grammar for each object into the Viterbi algorithm, which is used for HMM decoding. 
$88: 4 \cdot$ K. Ohara et al.

In summary, this study proposes a novel processing pipeline designed for recognizing the events of indoor objects using CSI. Our processing pipeline consists of (1) ICA-based blind source separation, (2) feature learningbased event recognition using convolutional recurrent neural networks (RNNs), and (3) correction of DNN estimates based on knowledge about the state transitions of an object using HMMs. Because CSI values are mixed signals affected by multiple objects or phenomena in an environment, we first decompose the mixed signals into independent sources. We then recognize the decomposed signals using the DNNs tailored for feature extraction from CSI data because intuitively designing features to be extracted from CSI data is difficult. Finally, we correct the sporadic errors of the estimates using HMMs, which incorporate knowledge about state transitions of an object.

The contributions of this paper are as follows. (1) To the best of our knowledge, this is the first study that attempts to detect the events of indoor everyday objects such as open/close events of doors and windows using Wi-Fi channel state information and deep learning. (2) We design an event recognition pipeline based on blind source separation using ICA, feature learning using a convolutional LSTM network, and knowledge-based error correction using HMMs. (3) We investigate the effectiveness of our method in real environments.

\section{RELATED WORK}

\subsection{Indoor event detection}

Many distributed sensing approaches employ a large number of small sensors such as switch sensors, RFID tags, vibration sensors, and accelerometers attached to daily objects [32, 40,41]. Although the distributed sensing approach can achieve fine-grained measurements of daily lives, its deployment and maintenance costs, e.g., battery replacement costs, are high. To cope with these problems, energy-harvesting sensors for building monitoring have been studied. For example, Campbell et al. [8] developed a vibration detector using a piezo-film vibration sensor that, when vibrated, generates current and transmits a packet announcing that the vibration event occurred. The sensor can be used to detect door open/close, window open/close, cabinet open/close, and refrigerator door open/close events.

Barometric pressure sensors have been used to detect door open/close events. Patel et al. [30] employ an HVAC air filter attached with pressure sensor units to detect pressure variation caused by door open/close events and room-to-room transitions. Wu et al. [48] employ a barometer in a smartphone to detect door open/close events based on the fact that, when a door in a building with HVAC systems is opened, a sharp change in indoor pressure can be observed. These approaches do not require sensors attached to doors. Shi et al. [37] employ FM-radio signal receivers to recognize the indoor situations "empty room," "opened door," and "walking person" based on the fact that changes in an environment impact the propagation of radio waves. In contrast, we employ commodity Wi-Fi APs and Wi-Fi modules. In addition, our method, which employs fine-grained CSI data, can detect the events of each individual door or window in a room.

WiSee [33] and WiTrack [3] achieve fine-grained device-free gesture recognition using Wi-Fi signals. However, these systems also require specialized USRP monitors for extracting signal features.

\subsection{Wi-Fi RSSI-based context recognition}

2.2.1 Indoor positioning. Many researchers have attempted to construct indoor positioning systems utilizing Wi-Fi RSSI. An advantage of Wi-Fi-based positioning is that we can use a smartphone with a Wi-Fi module as a signal receiver. For standard Wi-Fi-based positioning, fingerprinting techniques have usually been employed to measure indoor positions $[17,25,26]$. Fingerprinting employs a training phase in which Wi-Fi signals, i.e., the unique MAC addresses of APs and the received signal strengths from APs, are observed at known training coordinates. A set of APs and their signal strengths constitute a fingerprint that is unique to those coordinates.

Proceedings of the ACM on Interactive, Mobile, Wearable and Ubiquitous Technologies, Vol. 1, No. 3, Article 88. Publication date: September 2017. 
However, because standard Wi-Fi-based indoor positioning assumes that a user always possesses a smartphone, it is difficult to use this approach for such applications as monitoring an independently living elderly person or home automation. Therefore, device-free passive indoor positioning methods have recently been attracting attention [28, 31, 35, 49, 51]. With the device-free passive indoor positioning, a wireless sensor network is used to detect the movement of a person using the fact that radio frequency (RF) signals transmitted between a transmitter and receiver are affected by human movement. In [51], for example, the authors assume that Wi-Fi APs and Wi-Fi signal receivers are installed in an environment, and the receivers collect Wi-Fi RSSI fingerprints when a person is at each training point in a training phase.

2.2.2 Activity recognition. Wi-Fi RSSI has also been used to recognize human activities. Sigg et al. [38] proposed a device-free activity recognition method using a smartphone as a Wi-Fi receiver. The system uses smartphones, as the Wi-Fi receiver originally had limitations because RSSI information was not available per packet on Android smartphones. Hence, it was only possible to measure signal strength information as a per-second average $(1 \mathrm{~Hz})$, making it difficult to conduct Wi-Fi-based device-free activity recognition when using a standard Android smartphone. The authors solved this problem by modifying the Wi-Fi device driver to allow them to run the smartphone in monitor mode and then executed tcpdump to capture individual packets. Abdelnasser et al. [2] attempted to monitor breathing rates and patterns by employing the RSSI observed at a Wi-Fi enabled device such as a smart phone held on a person's chest to achieve non-invasive respiratory rate estimation and apnea detection.

\subsection{Wi-Fi CSI-based context recognition}

Commonly used Wi-Fi RSSI values are easily varied by the multipath effect. Moreover, because RSSI is measured from the RF signal at per-packet level and thus represents an aggregated scalar value at packet level, it is reportedly difficult to obtain an accurate signal condition [47]. In contrast to RSSI, multiple CSIs can be obtained at one time and are fine-grained values obtained from the PHY layer that describe the amplitude and phase information on each subcarrier and for each transmit and receive antenna in MIMO. Therefore, context recognition methods leveraging Wi-Fi CSI data have been actively studied.

2.3.1 Channel state information. In a wireless network, the channel quality between the transmitter and receiver, which is extracted from the PHY layer, is known as CSI. CSI describes the combined effect of, for example, the scattering, fading, Doppler shift, and power decay of the transmitted signal. In OFDM systems, e.g., $802.11 \mathrm{a} / \mathrm{g} / \mathrm{n}$ networks, data are transmitted using orthogonal subcarriers at different frequencies. In a narrow-band flat-fading channel, a MIMO system can be modeled as

$$
\boldsymbol{Y}_{i}=\boldsymbol{H}_{i} \boldsymbol{X}_{i}+\boldsymbol{N}_{i},
$$

where $\boldsymbol{X}_{i}$ and $\boldsymbol{Y}_{i}$ are the $M_{T}$-dimensional transmitted signal vector and $M_{R}$-dimensional received signal vector for the $i$ th subcarrier, respectively. Note that $M_{T}$ is the number of transmit antennas and $M_{R}$ is the number of receiver antennas in an $M_{T} \times M_{R}$ MIMO system. In addition, $\boldsymbol{N}_{i}$ represents an $M_{R}$-dimensional noise vector and $\boldsymbol{H}_{i}$ is the $M_{T} \times M_{R}$ dimensional channel matrix and is known as the CSI for the $i$ th subcarrier. Matrix $\boldsymbol{H}_{i}$ can be represented as

$$
\boldsymbol{H}_{i}=\left[\begin{array}{cccc}
h_{11} & h_{12} & \ldots & h_{1 M_{R}} \\
h_{21} & h_{22} & \ldots & h_{2 M_{R}} \\
\vdots & \vdots & \ddots & \vdots \\
h_{M_{T} 1} & h_{M_{T} 2} & \ldots & h_{M_{T} M_{R}}
\end{array}\right],
$$

where $h_{m n}$ is the CSI for the $m$ th transmitter antenna and $n$th receiver antenna pair. Note that $h_{m n}$ is a complex value and represented by

$$
h_{m n}=\left\|h_{m n}\right\| e^{j \angle h_{m n}},
$$


88:6 - K. Ohara et al.

where $\angle h_{m n}$ is the phase of $h_{m n}$. When we have $N_{S}$ subcarriers, we can obtain $N_{S}$ channel matrices

$$
\boldsymbol{H}=\left[\boldsymbol{H}_{1}, \boldsymbol{H}_{2}, \ldots, \boldsymbol{H}_{N_{S}}\right] .
$$

Channel matrix $\boldsymbol{H}$ is extractable for each packet from commodity Wi-Fi network interface cards such as the Intel 5300 NIC.

2.3.2 Indoor positioning. We first introduce indoor positioning studies locating a CSI-enabled portable device. Sen et al. [36] use complex CSI vectors for each subcarrier to construct location clusters in a training phase and compute the cross-correlation between test CSI data and each cluster to locate a portable device. Wu et al. [47] aggregate $M_{T} \times M_{R} \times N_{S}$ CSI data into one single value that reflects the estimated distance between a transmitter and receiver ( $M_{T}=M_{R}=1$ in their system). Therefore, the value is computed for each observed AP, and the authors construct a CSI fingerprint concatenating the computed values. Chapre et al. [10] extract amplitude and phase information from channel matrix $\boldsymbol{H}$ to construct a CSI-MIMO fingerprint for indoor localization.

As for CSI-based device-free passive localization, Abdel-Nasser et al. [1] install a Wi-Fi AP and a signal receiver in a room to locate a person who does not possess any portable devices. The authors employ CSI magnitudes to form location clusters based on the k-means algorithm using training data collected varying the position of a person in the room, and compare test CSI data with the constructed cluster to locate a person. E-eyes [46] achieves device-free location-oriented activity recognition using Wi-Fi CSI. The system predicts a user's activities such as cooking and eating based on the estimated indoor locations of the user. To construct activity/location fingerprints, the system employs a clustering algorithm to identify similar instances of activities.

2.3.3 Activity recognition. Wi-Fi CSI has also been used to achieve device-free activity recognition. Wang et al. [45] attempt to detect primitive actions using CSI and estimate activities consisting of primitive actions using multi-class support vector machines. In [42], the authors propose two models for quantitatively correlating CSI dynamics and human activities; a CSI-speed model that correlates CSI value dynamics and human movement speeds, and a CSI-activity model that correlates the movement speeds of different human body parts and a specific activity.

Device-free fall detection for elder care support is a typical application of Wi-Fi CSI. WiFall [19] employs the time variability and spatial diversity of CSI to detect falls in residential settings. Anti-Fall [53] employs the CSI phase difference over two antennas and uses amplitude information to distinguish the fall activity from fall-like activities.

In [27], the authors attempt to capture a user's sleep information such as respiration based on Wi-Fi CSI by extracting rhythmic patterns associated with respiration. Ali et al. [4] attempt to recognize keystrokes by extracting a unique pattern in the time-series of CSI values for each key.

\subsection{Context recognition using deep learning}

The above mentioned CSI-based context recognition studies using machine learning employ handcrafted features designed by researchers. In contrast, our study employs a feature learning approach using DNNs. Here we introduce studies on context recognition using deep learning. Wang et al. [44] apply deep learning to CSI-based indoor positioning using a neural network with four hidden fully connected layers. They also extract calibrated phase information from CSI data to train a network with three hidden fully connected layers for indoor positioning [43]. Zeng et al. [52] employ a neural network consisting of one convolutional layer and two fully connected layers for activity recognition using a wrist-worn three-axis accelerometer. Yang et al. [50] use a neural network consisting of four convolutional layers and one fully connected layer to evaluate the Opportunity data set [11]. Similar to us, Ordóñez et al. [29] employ three convolutional layers and two LSTM layers for acceleration-based activity recognition. Because they focus on accelerometers attached to different body parts, they perform onedimensional convolution along the time axis for each one-dimensional sensor data stream. In contrast, we design

Proceedings of the ACM on Interactive, Mobile, Wearable and Ubiquitous Technologies, Vol. 1, No. 3, Article 88. Publication date: September 2017. 
Detecting State Changes of Indoor Everyday Objects using Wi-Fi Channel State Information • 88:7

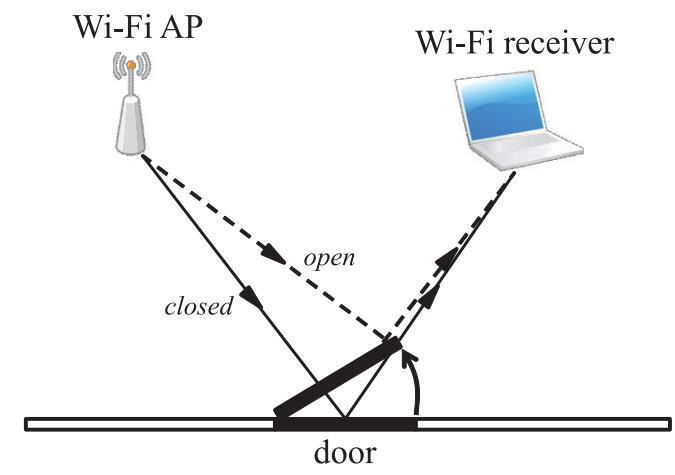

Fig. 2. Illustration of the impact of door state change on signal propagation

convolutional layers tailored for multichannel CSI data that enable us to extract meaningful features based on the findings of prior CSI studies.

\section{PROPOSED METHOD}

\subsection{Overview}

We assume that a commodity Wi-Fi AP and a computer or sensor node with a commodity Wi-Fi module such as the Intel 5300 NIC are installed in a room. We obtain time-series CSI data from the NIC and detect indoor events such as door open/close and window open/close using the time-series data. For example, when a door in a room opens, the event alters a signal reflection path, as shown in Fig. 2. To detect such indoor events, we extract and analyze CSI data that describe how a signal propagates from a transmitter to a receiver. Note that we attempt to estimate an event/state of an indoor object at each time slice. For example, a door can have "open" and "close" events and "opened" and "closed" states. Therefore, we estimate which state or event the door is in at each time slice. In addition, similar to many other CSI-based context recognition studies, we assume that we can detect events that occur in a room where the transmitter and receiver are placed. We assume that there are several everyday objects such as doors, windows, and cabinets in the room, and an event/state recognition architecture shown in Fig. 3 is prepared for each object.

Our method first preprocesses CSI signals using blind source separation. The existing CSI-based context recognition studies preprocess raw CSI signals according to their purposes, and recent studies have used both the amplitude and phase information of CSI signals. In addition to the amplitude and phase time series, we decompose CSI signals, i.e., $M_{T} \times M_{R} \times N_{S}$ dimensional time-series data, into independent signals using ICA, and employ the decomposed independent signals as the DNN input. Next, the original amplitude, original phase, decomposed amplitude, and decomposed phase time-series data are fed into a DNN. The classification results of the network are then input into HMMs to capture the temporal regularity of physical events.

The classification approaches used in machine learning are divided into two groups: one group uses discriminative techniques that learn the class boundaries and the other uses generative techniques that model the conditional density functions of the classes. The classification performances of the discriminative techniques, which find discriminant features of the classes, often outperform those of generative techniques. In contrast, handling missing data is often easier with the generative techniques. In addition, because we deal with time-series data, incorporating an HMM, which is a generative model that can be used to model events/states with temporal patterns,

Proceedings of the ACM on Interactive, Mobile, Wearable and Ubiquitous Technologies, Vol. 1, No. 3, Article 88. Publication date: September 2017. 


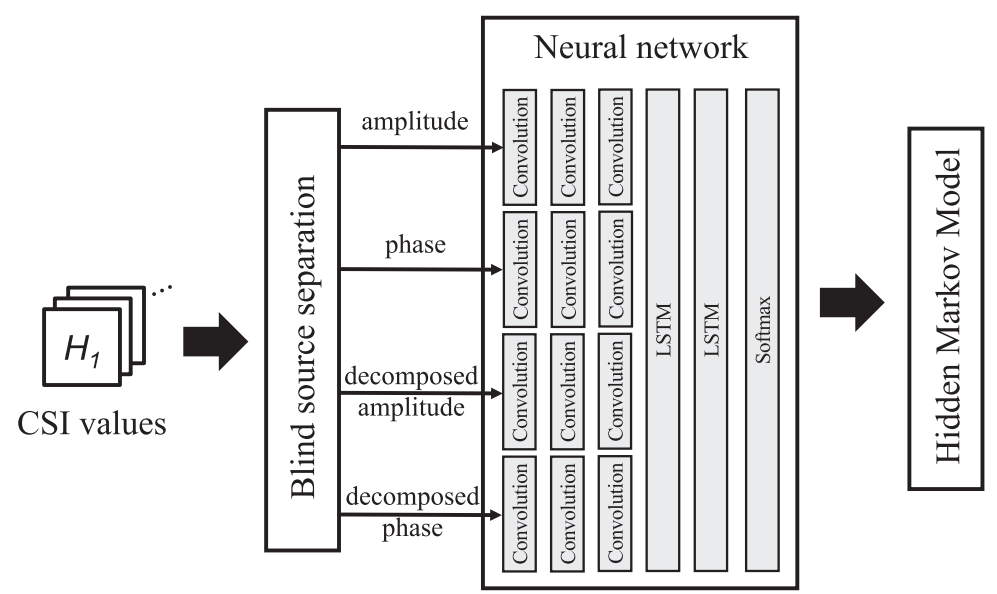

Fig. 3. Our proposed event recognition pipeline for indoor event detection using CSI

can improve the performance and smoothness of the event/state recognition. This study employs a hybrid generative/discriminative classifier consisting of DNNs and HMMs to utilize the advantages of both discriminative classifiers and generative models.

Because our method relies on supervised machine learning techniques, it consists of training and test phases. In the training phase, labeled training data are used to compute an unmixing matrix tailored to an object of interest, which is detailed later, and we train the DNN and HMMs. In the test phase, we classify an unlabeled sensor data segment within each sliding time window into an appropriate class using the trained network and HMMs.

\subsection{Blind source separation using ICA}

As mentioned in the related work section, $\left(M_{T} \times M_{R} \times N_{S}\right)$-dimensional CSI time-series data are obtained. From each one-dimensional time-series data, we extract the amplitude time-series data and phase time-series data, i.e., the time-series data of $\left\|h_{m n}\right\|$ and $\angle h_{m n}$. We decompose the amplitude and phase time series using ICA and use the decomposed signals in addition to the original signals as DNN input. Because the numbers of dimensions of the input and output data of ICA are identical in our setting, $M_{T} \times M_{R} \times N_{S}$ dimensional decomposed time-series data are obtained from CSI amplitude (or phase) values. As above, $4 \times M_{T} \times M_{R} \times N_{S}$ time-series data are extracted from the raw CSI time series.

Blind source separation is a method for separating mixed source signals without using information about the nature of the signals and has been studied in the speech recognition research area. Assume a party with several simultaneous conversations and with several microphones located at different places in a room, simultaneously recording the conversations. The aim of blind source separation in this scenario is to separate the mixed microphone signals into each independent conversation. Each microphone signal can be modeled as a linear mixture of the source signals by

$$
\boldsymbol{x}(t)=\boldsymbol{A s}(t),
$$

where $\boldsymbol{x}(t)=\left[x_{1}(t), x_{2}(t), \ldots, x_{M}(t)\right]^{T}$ represents the measurements of the microphones at time $t$. In addition, $\boldsymbol{s}(t)=\left[s_{1}(t), s_{2}(t), \ldots, s_{M}(t)\right]^{T}$ represents the source signals. Further, $M$ is the number of microphones and $\boldsymbol{A}$ is an $M \times M$ mixing matrix that mixes the source signals. Therefore, the objective of blind source separation is to estimate unmixing matrix $\boldsymbol{W}$ and compute $\boldsymbol{s}(t)$ using $\boldsymbol{W}$ and $\boldsymbol{x}(t)$ by

$$
\boldsymbol{s}(t)=\boldsymbol{W} \boldsymbol{x}(t) .
$$

Proceedings of the ACM on Interactive, Mobile, Wearable and Ubiquitous Technologies, Vol. 1, No. 3, Article 88. Publication date: September 2017. 
Detecting State Changes of Indoor Everyday Objects using Wi-Fi Channel State Information • 88:9
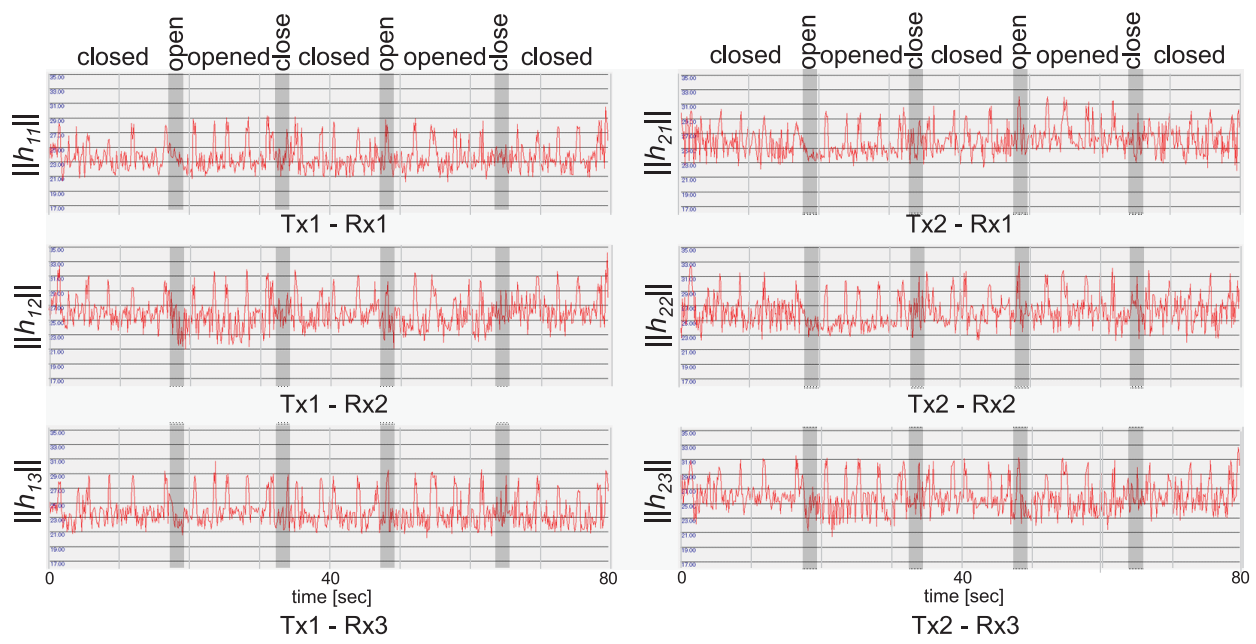

Fig. 4. Raw CSI time-series data for a certain subcarrier obtained when the "open" and "close" events of a door occurred. We cannot find apparent changes in the signals when events occurred.
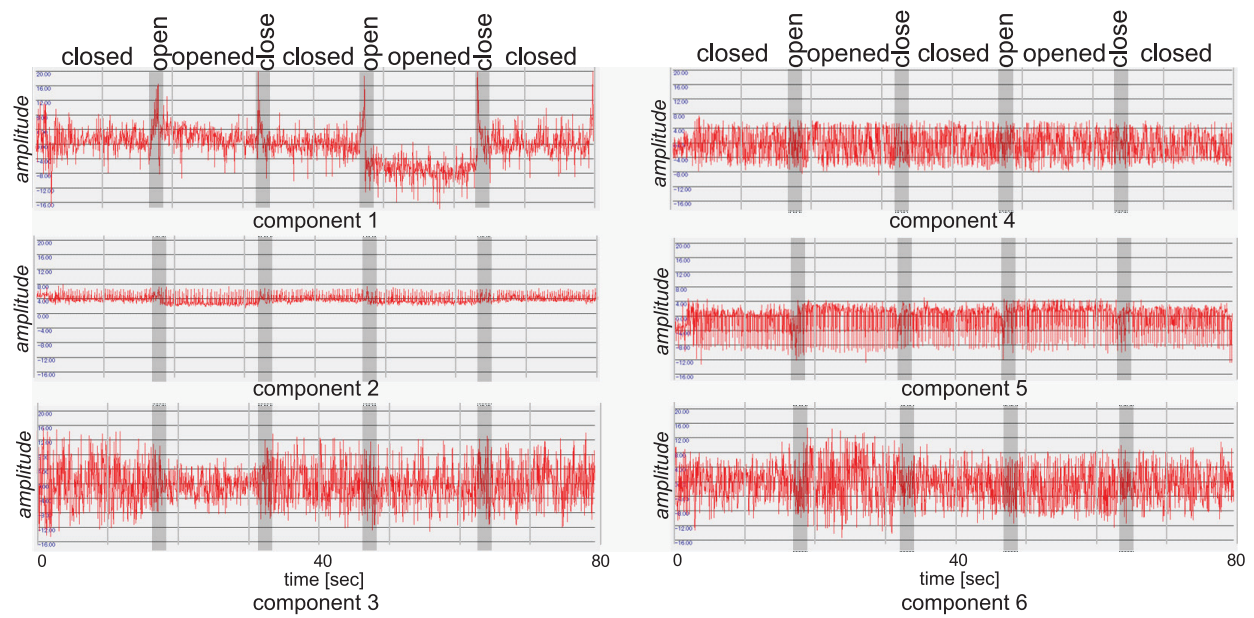

Fig. 5. Decomposed signals obtained from raw signals shown in Fig. 4 by ICA. Component 1 captures signal changes when events occurred.

Note that, because the number of speakers (objects and other events that affect radio propagation in our case) is unknown, we simply use an $M \times M$ mixing matrix, i.e., the number of source signals and output signals are identical. Our experiment uses 180 input signals $\left(M_{T}=2, M_{R}=3\right.$, and $\left.N_{S}=30\right)$, and this number can be sufficiently larger than the number of objects in an environment.

ICA [14] has been used to compute $\boldsymbol{W}$ in blind source separation. ICA separates source signals relying on the assumption that the source signals are mutually statistically independent. This is not an unrealistic assumption in our case because indoor everyday objects such as doors and windows are not always simultaneously used (e.g., opened). In this study, we use FastICA [20] to compute $\boldsymbol{W}$. The ICA algorithm first whitens an observed data 
matrix by projecting the data onto its principal component directions, and then the algorithm estimates $\boldsymbol{W}$. Matrix $\boldsymbol{W}$ is chosen to maximize the negentropy approximation under the constraints that $\boldsymbol{W}$ is an orthonormal matrix, ensuring that the estimated components are independent. For more detail about the algorithm, see [20].

To capture minute changes in signals caused by an object of interest, we construct an unmixing matrix tailored to the object. Our approach is simple. From labeled training data, we extract segments related to events of the object and use only the extracted segments to compute $\boldsymbol{W}$ tailored to the object. For example, when a start time and end time of an "open" event is $t_{s}$ and $t_{e}$, respectively, we extract the segment between $t_{s}-\Delta$ and $t_{e}+\Delta$. With such segments, we can obtain a $\boldsymbol{W}$ that focuses more on the events of the object. This approach also permits us to reduce the computation time of the ICA algorithm because we use only segments corresponding to events of the object.

Figures 4 and 5 show an example of blind source separation using ICA. Fig. 4 shows raw CSI time-series data for a certain subcarrier obtained when the "open" and "close" events of a door occurred. Because $M_{T}=2$ and $M_{R}=3$, we can obtain six-dimensional CSI time-series data for each subcarrier. As shown in the figure, we cannot find apparent changes in the signals. Fig. 5 shows the decomposed source signals computed by ICA. We can find sudden increases in the first time-series data (component 1) of Fig. 5, which correspond to the "open" and "close" events.

\subsection{Deep neural network}

3.3.1 Network architecture. As shown in Fig. 3, our network consists of three convolutional layers, two LSTM layers, and one softmax output layer. The number of layers is determined based on our preliminary experiment. Note that the convolutional layers are prepared for the amplitude and phase information extracted from the decomposed CSI in addition to the amplitude and phase information of the raw CSI. Because we employ a sliding window model with $80 \%$ overlap, the inputs of the first convolutional layer are a time-series of amplitude (or phase) values within a window at time $t$ whose width is $W_{T}$ (500 samples corresponding to $0.5 \mathrm{~s}$ ). For example, for the raw amplitude information, the inputs are a time-series of 180-dimensional $\left(M_{T} \times M_{R} \times N_{S}\right)$ amplitude values, and the length of the time-series is $W_{T}$. The convolutional layers are used for feature extraction and the LSTM layers are used for recognizing the time-series of the third convolutional layer outputs. The convolutional layers are designed to extract meaningful features from the multichannel CSI data based on the findings of existing studies on CSI. The output layer using softmax function is used to output class probabilities (scores, to be exact) for the window at time $t$. For example, class probabilities for "open," "close," "opened," and "closed" are computed.

3.3.2 Convolutional layers. Figure 6 shows an overview of the convolutional layers of our network. (This is an example of raw CSI amplitude data.) The convolutional layers have been used to learn efficient filters (kernels) for feature extraction in the image classification task [12].

As mentioned above, an input of the first convolutional layer is a $500 \times 180$ matrix. Note that 500 corresponds to the number of time slices and 180 corresponds to the number of dimensions for each time slice. As shown in the left portion of Fig. 6, the 180-dimensional data consist of $M_{T} \times M_{R}=6$ values (amplitude or phase) for each subcarrier. For example, a $500 \times 6$ red-colored matrix is prepared for $H_{1}$ in the left portion of Fig. 6. Because the number of subcarriers is $N_{s}=30$, we have 180 -dimensional data $(6 \times 30)$ for each time slice. Also, sets of CSI values for 30 subcarriers (e.g., $H_{1}, H_{2}$, and $H_{3}$ ) are sorted in the order of their frequencies. In the first convolutional layer, we extract the features from each subcarrier, i.e., by convolving values from different MIMO channels on the same subcarrier, using an $F_{t} \times N_{p}$ filter $\left(F_{t}=100\right.$ and $\left.N_{p}=M_{T} \times M_{R}=6\right)$. We use a stride (step size) of 1 sample in terms of the time axis (the horizontal direction of the input in Fig. 6). In addition, we use a stride of $6\left(M_{T} \times M_{R}=6\right)$ in terms of the vertical axis to convolve values from the same subcarriers because the CSI data in each subcarrier are reportedly correlated with each other [10]. With this filter, we can obtain a $400 \times 30$ output matrix (30 is the number of subcarriers).

Proceedings of the ACM on Interactive, Mobile, Wearable and Ubiquitous Technologies, Vol. 1, No. 3, Article 88. Publication date: September 2017. 
Detecting State Changes of Indoor Everyday Objects using Wi-Fi Channel State Information • 88:11

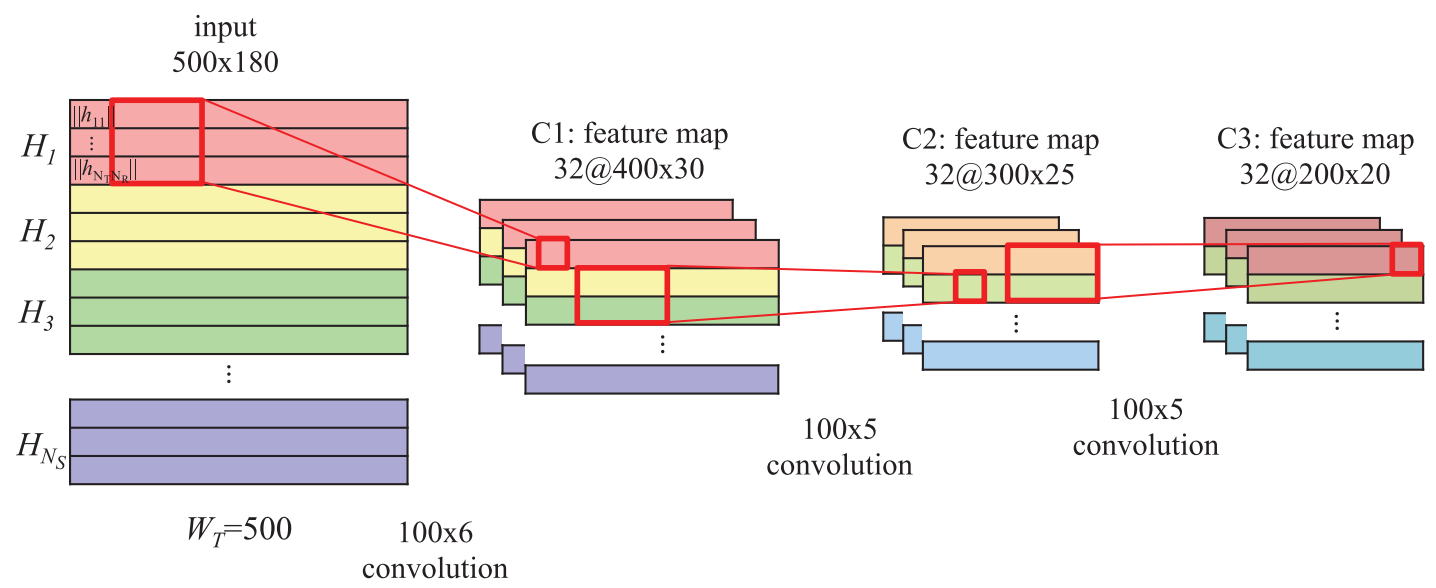

Fig. 6. Convolutional layers in our network

In the second layer, we extract features by convolving information from adjacent subcarriers using an $F_{t} \times N_{f}$ filter $\left(N_{f}=5\right)$ because subcarriers with similar frequencies are reported to correlate with each other [47]. We use a stride (step size) of 1 in the horizontal and vertical directions. We also use the same filter in the third layer. These convolutional layers employ a rectified linear units (ReLU) activation function, and the number of filters of each convolutional layer is 32 . As above, we employ the stack of the convolutional layers designed for CSI data to extract features.

3.3.3 LSTM layers. We employ the recurrent layers (LSTM layers) to learn the temporal dynamics of the features extracted by the convolutional layers. LSTM is a RNN architecture with memory cells, and it permits us to learn temporal relationships at a long time scale. A standard RNN fails to learn long-term dependencies because of the vanishing gradient problem [6]. LSTM solves this problem by employing memory cells that hold past information, updating the cell state using write, read, and reset operations with input, output, and forget gates [15]. Therefore, LSTM has been used to recognize time-series data, e.g., speech recognition and sentence classification [16, 54].

The output of each convolutional layer is a $200 \times 20 \times 32$ tensor, where 200 corresponds to time. Therefore, a 2,560 -dimensional vector $(20 \times 32 \times 4=2560)$ is fed into the fourth layer (LSTM layer) at each time slice. (Remember that we prepare convolutional layers for four inputs: raw amplitude, raw phase, decomposed amplitude, and decomposed phase.) An output of the fourth layer at each time slice is then fed into the fifth layer. These LSTM layers employ a sigmoid activation function and, the number of nodes of each LSTM layer is 128. Because we believe that an output at the last time slice can hold information about the entire time-series within the window, only an output at the last time slice of the fifth layer is fed into the softmax output layer, which is used to output class probabilities in a multiclass classification.

3.3.4 Training network. We train the network to minimize the cross-entropy between a distribution of ground truth and an estimated distribution by the softmax output layer, employing backpropagation based on Adam [23], which enables us to automatically adjust the learning rate. (The learning rate is a parameter that controls the rate at which parameters are updated in a neural network.) In addition, to reduce overfitting, we employ dropout, which is a simple regularization technique in which randomly selected neurons are ignored during training [39].

For the convolutional layers, we employ batch normalization, enabling us to prevent model divergence and increase convergence speeds through higher learning rates by normalizing outputs of the layers [21]. When we 
$88: 12 \cdot$ K. Ohara et al.

train the network, training data are divided into mini-batches. In the batch normalization, the outputs of each neuron are normalized for each mini-batch, i.e., subtracting the mean and then dividing by the standard deviation. Here, our task suffers from the class imbalance problem because the amount of training instances belonging to "opened" and "closed" states is much larger than that of the "open" and "close" events because the durations of the states are naturally much longer that those of the events. Therefore, we simply undersample instances belonging to the "opened" and "closed" states in each mini-batch to balance the data in the mini-batch using random undersampling.

Note that, when we recognize test data that are inherently imbalanced, the distribution of outputs of each neuron for the test data deviates from that for the training data because the training data were balanced, having a negative effect on the batch normalization. In standard batch normalization, in the training phase, outputs (activations) of the convolutional layers are normalized for each batch using the mean and standard deviation computed using the outputs of the training data. In the test phase, the outputs of the convolutional layers are normalized using the mean and standard deviation computed using the outputs of the test data. However, because the test data are unbalanced, the test data are normalized using a mean and standard deviation that are far different from those of the training data. Our solution for this issue is to use the mean and standard deviation obtained in the training phase to normalize the neuron outputs for the test data.

\subsection{HMM decoding with grammar}

As mentioned in Section 3.1, a vector consisting of class probabilities is generated by the DNN for each time window. We then employ HMMs to recognize events/states related to the object of interest using the probability vector sequence.

3.4.1 Recognition with HMMs. A sequence of class probability vectors is input into the HMMs. A leftto-right HMM is prepared for each event/state of the object, e.g., "open," "close," "opened," and "closed" for a door, where the values of the observed variables correspond to the probability vectors, and we represent its output distributions using Gaussian mixture densities (Gaussian mixture model: GMM). The hidden variable of the HMM corresponds to the internal state of the event. In the model, the hidden variable at time $t$ depends only on the previous hidden variable at time $t-1$. In addition, the observed variable at time $t$ depends only on the hidden variable at time $t$. Therefore, using the HMMs allows us to capture the temporal regularity of events. We employ the Baum-Welch algorithm [34] to estimate the HMM parameters.

When we recognize test data (probability vector sequence) using the trained HMMs, we use the Viterbi algorithm to find the most probable state sequence in/across the HMMs [34]. From the state sequence, we can know into which HMM (event/state) a probability vector at time $t$ is classified.

3.4.2 Decoding with grammars. Because a left-to-right HMM is prepared for each event/state, the Viterbi algorithm finds state transitions across the HMMs. This means that we take into account a state transition from the last state of an arbitrary HMM to the first state of another HMM. With HMMs for a door, for example, this corresponds to a state transition from a "closed" HMM to an "open" HMM (and to all other HMMs of the door). By taking the above state transition into account, we can represent transitions of events. Here, we can specify state transitions among HMMs using a handcrafted grammar based on prior knowledge about an object of interest. With HMMs for a door, for example, we can specify that an "open" event occurs only when the door is in a "closed" state. We construct such a grammar for each object and investigate its effect in the evaluation section.

We provide a grammar example written in extended BNF. The grammar for a door is described as follows.

Proceedings of the ACM on Interactive, Mobile, Wearable and Ubiquitous Technologies, Vol. 1, No. 3, Article 88. Publication date: September 2017. 
Detecting State Changes of Indoor Everyday Objects using Wi-Fi Channel State Information • 88:13

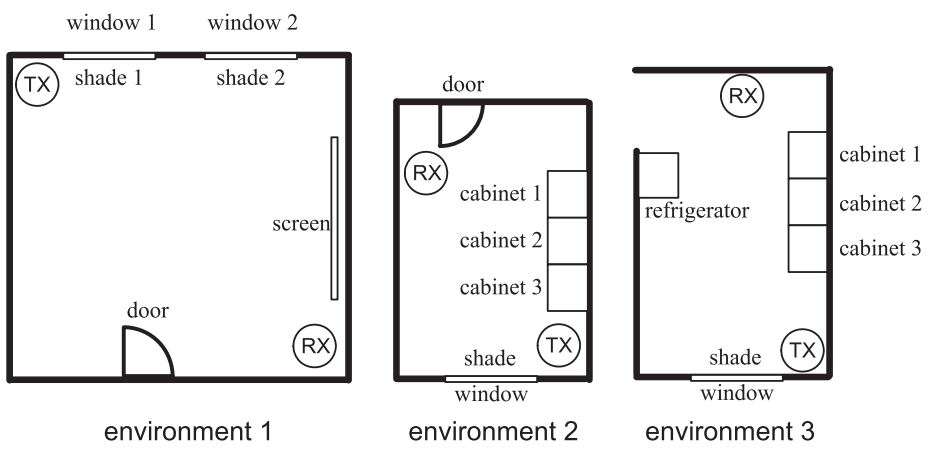

Fig. 7. Three experimental environments. $T X$ and $R X$ indicate the transmitter and receiver, respectively. The sizes of environments 1, 2, and 3 are $7.2 \mathrm{~m} \times 10.2 \mathrm{~m}, 3.5 \mathrm{~m} \times 7.5 \mathrm{~m}$, and $3.5 \mathrm{~m} \times 9.5 \mathrm{~m}$, respectively.

[opened close] \{closed open opened close $\}$ closed [open opened]

We first focus on " $\{$ closed open opened close\}." With this expression, we can define that "closed," "open," "opened," and "close" events/states occur sequentially and are repeated because " \{\} " shows a repetition. More specifically, for example, the transition probabilities from the last state of the "closed" HMM to the first states of all other HMMs except for the "open" HMM are defined as zero. In contrast, the transition probability to the "open" HMM is one.

We next explain "[opened close]." This expression represents that the possible initial state of the door in the given test data is the "opened" or "closed" state because "[ ]" represents an option and the "closed" state is specified in the beginning of "\{closed open opened close $\}$." The "close" event in "[opened close]" describes that the "close" event occurs between the "opened" and "closed" states. As above, we design the grammar so that our method works even when the initial state of the door is not given. Similarly, "closed [open opened]" represents that the possible last state is the "opened" or "closed" state.

As aforementioned, we prepared a single left-to-right HMM for each event/state. In addition, a grammar was prepared separately. This is because we want to explicitly specify the state transitions between multiple events. Because the number of event/state transitions is much smaller than the number of feature vectors in general, it is difficult to learn the transition probability across the events/states. With this setting, we can incorporate knowledge about the event/state transitions with no training data related to event/state transition.

\section{EVALUATION}

\subsection{Data set}

We collected sensor data in real three environments. Fig. 7 shows our three experimental environments and their settings. In environments 1 and 2, we recognize the events of commonly used daily objects such as doors and windows. In environments 2 and 3, we try to recognize the events of adjacent storage cabinets, which may exhibit similar sensor data. Environment 1 includes eight tables and 20 chairs, which are not depicted in Fig. 7. Environment 2 also includes one desk, one table, and one chair. Environment 3 includes one table and eight chairs.

We installed a Wi-Fi AP (Buffalo WXR-2533DHP) and a PC with the Intel 5300 NIC in each environment as shown in Fig. 7. A modified NIC driver developed by Halperin et al. [18] was installed on the PC to collect CSI 
Table 1. Objects used in the three environments

\begin{tabular}{|c|c|c|}
\hline environment 1 & environment 2 & environment 3 \\
\hline \hline door & door & window \\
\hline window 1 & window & window shade \\
\hline window shade 1 & window shade & cabinet 1 \\
\hline window 2 & cabinet 1 & cabinet 2 \\
\hline window shade 2 & cabinet 2 & cabinet 3 \\
\hline pull down projector screen & cabinet 3 & refrigerator \\
\hline
\end{tabular}

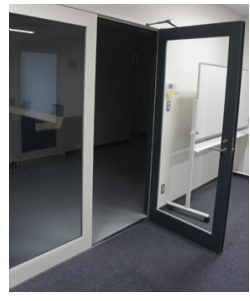

door

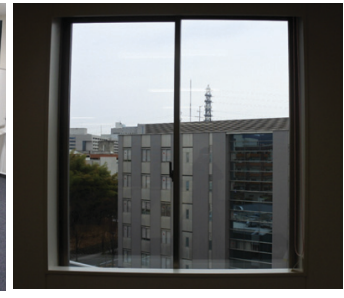

window

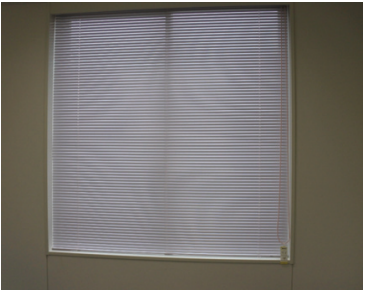

window shade

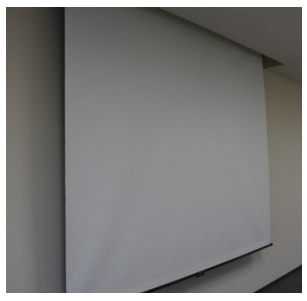

projector screen

Fig. 8. Examples of objects used in environment 1

data. We use the $\mathrm{AP}$ as a transmitter and the $\mathrm{PC}$ as a receiver. The transmitter has two antennas and the receiver has three antennas, i.e., $M_{T}=2$ and $M_{R}=3$. In addition, the number of subcarriers is 30 , i.e., $N_{S}=30$. We measured the CSI values using udp packets sent from the transmitter to the receiver at a rate of approximately $1,000 \mathrm{~Hz}$.

In each environment, a participant conducted 150 sessions of data collection. Throughout a session, the participant used all objects so that each event of the objects occurred once in an arbitrary order. That is, in a session, for example, a door can be opened while a window can be closed. In another session, the door can be closed while the window is opened. As above, each object is used under different conditions in our experiment. Table 1 lists the objects used in each environment, and Fig. 8 shows photographs of the objects used in environment 1. In addition, Table 2 lists the events and states of the objects. Each object has two events and two states, e.g., "open" and "close" events and "opened" and "closed" states for a door. Because the participant walked at random in the room and used the objects in a random sequence, the objects such as doors were used from different sides and in different positions. To include noise caused by a person living in the environment in our sensor data, the participant also walked around at random in each session. The duration of "open" and "close" events for doors and windows was about $3 \mathrm{~s}$. In addition, the duration of "open" and "close" events for window shades was about $6 \mathrm{~s}$. To obtain ground truth, we recorded the sessions with a video camera.

Tables 4, 5, and 6 show the number of time windows (instances) for each event/state included in our labeled sensor data. As shown in the tables, the numbers of instances for events are much smaller than those for states.

\subsection{Evaluation methodology}

We evaluated the performance of our method for each environment. We randomly selected $90 \%$ of the sessions as training sessions and used the remaining sessions as test sessions. We prepared the following methods to investigate the effectiveness of the ICA, convolutional layers, grammars, and HMMs. In addition to these methods, 
Detecting State Changes of Indoor Everyday Objects using Wi-Fi Channel State Information • 88:15

Table 2. Events and states of objects. Bold face indicates events and italics indicates states.

\begin{tabular}{|c|c|}
\hline object & events/states \\
\hline \hline door & open, close, opened, closed \\
\hline window & open, close, opened, closed \\
\hline window shade & open, close, opened, closed \\
\hline $\begin{array}{c}\text { pull down } \\
\text { projector screen }\end{array}$ & $\begin{array}{c}\text { pull-down, retract, } \\
\text { pulled-down, retracted }\end{array}$ \\
\hline cabinet & open, close, opened, ,losed \\
\hline refrigerator & open, close, opened , closed \\
\hline
\end{tabular}

Table 4. \# of time windows (instances) for each event/state in environment 1

\begin{tabular}{|c|c|c|c|c|}
\hline & \multicolumn{2}{|c|}{ event } & \multicolumn{2}{c|}{ state } \\
\hline & open & close & opened & closed \\
\hline \hline door & 5748 & 5712 & 97432 & 113478 \\
\hline window 1 & 5106 & 5269 & 60025 & 151970 \\
\hline window 2 & 5740 & 5736 & 55086 & 155808 \\
\hline shade 1 & 11760 & 11146 & 120528 & 78936 \\
\hline shade 2 & 11670 & 11209 & 112710 & 86781 \\
\hline screen & 6143 & 5675 & 92045 & 118507 \\
\hline
\end{tabular}

Table 3. Experimental parameters used in this study

\begin{tabular}{|c|c|}
\hline parameter & value \\
\hline \hline size of sliding window [sample] & 500 \\
\hline overlap of sliding window & $80 \%$ \\
\hline \# of states in HMM & 50 \\
\hline \# of Gaussians in GMM & 10 \\
\hline mini-batch size [session] & 50 \\
\hline
\end{tabular}

Table 5. \# of time windows (instances) for each event/state in environment 2

\begin{tabular}{|c|c|c|c|c|}
\hline & \multicolumn{2}{|c|}{ event } & \multicolumn{2}{c|}{ state } \\
\hline & open & close & opened & closed \\
\hline \hline door & 6135 & 7263 & 77064 & 87847 \\
\hline window & 6204 & 5670 & 46004 & 120431 \\
\hline shade & 11222 & 11932 & 95449 & 59706 \\
\hline cabinet 1 & 5621 & 5515 & 77721 & 89452 \\
\hline cabinet 2 & 6506 & 6577 & 78921 & 86305 \\
\hline cabinet 3 & 4969 & 5314 & 76173 & 91853 \\
\hline
\end{tabular}

Table 6. \# of time windows (instances) for each event/state in environment 3

\begin{tabular}{|c|c|c|c|c|}
\hline & \multicolumn{2}{|c|}{ event } & \multicolumn{2}{c|}{ state } \\
\hline & open & close & opened & closed \\
\hline \hline window & 6085 & 5582 & 48013 & 134245 \\
\hline shade & 13975 & 14878 & 107030 & 58042 \\
\hline cabinet 1 & 4720 & 5171 & 85610 & 98424 \\
\hline cabinet 2 & 4657 & 5222 & 90021 & 94025 \\
\hline cabinet 3 & 5035 & 5475 & 85027 & 98388 \\
\hline refrigerator & 4402 & 3833 & 69855 & 115835 \\
\hline
\end{tabular}

we prepared a method that employs a dimensionality reduction method and handcrafted features used in state-ofthe-art studies.

- Proposed: This is our proposed method. The structure of the grammar for each object is identical to that for the door described in the previous section.

- w/o ICA: This method does not use time-series data obtained by ICA. It simply uses amplitude and phase time-series data extracted from the raw CSI data.

- w/o Conv: This method does not use the convolutional layers. Therefore, raw amplitude, raw phase, decomposed amplitude, and decomposed phase data are directly fed into the LSTM layer.

- w/o grammar: This method does not use a handcrafted grammar for each object. That is, the transition probabilities from one HMM to the other HMMs are uniform.

- w/o HMM: This method does not use HMMs. We simply use the classification results of the output layer. 
$88: 16 \cdot$ K. Ohara et al.

Table 7. Classification accuracies for Proposed in environment 1

\begin{tabular}{|c||c|c|c|}
\hline & Precision & Recall & F-measure \\
\hline \hline door & 0.832 & 0.921 & 0.870 \\
\hline window 1 & 0.824 & 0.930 & 0.870 \\
\hline window 2 & 0.816 & 0.939 & 0.866 \\
\hline shade 1 & 0.867 & 0.921 & 0.891 \\
\hline shade 2 & 0.824 & 0.870 & 0.834 \\
\hline screen & 0.791 & 0.892 & 0.832 \\
\hline average & 0.826 & 0.912 & 0.861 \\
\hline
\end{tabular}

Table 8. Classification accuracies for Proposed in environment 2

\begin{tabular}{|c||c|c|c|}
\hline & Precision & Recall & F-measure \\
\hline \hline door & 0.838 & 0.932 & 0.877 \\
\hline window & 0.787 & 0.960 & 0.849 \\
\hline shade & 0.790 & 0.827 & 0.804 \\
\hline cabinet 1 & 0.796 & 0.939 & 0.849 \\
\hline cabinet 2 & 0.811 & 0.930 & 0.855 \\
\hline cabinet 3 & 0.794 & 0.925 & 0.844 \\
\hline average & 0.803 & 0.919 & 0.846 \\
\hline
\end{tabular}

Table 9. Classification accuracies for Proposed in environment 3

\begin{tabular}{|c||c|c|c|}
\hline & Precision & Recall & F-measure \\
\hline \hline window & 0.836 & 0.967 & 0.888 \\
\hline shade & 0.918 & 0.930 & 0.924 \\
\hline cabinet 1 & 0.795 & 0.925 & 0.846 \\
\hline cabinet 2 & 0.825 & 0.961 & 0.879 \\
\hline cabinet 3 & 0.823 & 0.958 & 0.877 \\
\hline refrigerator & 0.734 & 0.919 & 0.795 \\
\hline average & 0.822 & 0.943 & 0.868 \\
\hline
\end{tabular}

- $R F$ : This method first applies PCA on the raw amplitude and phase data within a window and then extracts the mean, variance, kurtosis, skewness, maximum, minimum, and difference between the maximum and minimum features. Next, the method constructs a feature vector concatenating the features and classifies the vector into an appropriate class using the random forest classifier [7].

- HMM: This method first applies PCA on the raw amplitude and phase data within a window and then extracts the mean, variance, kurtosis, skewness, maximum, minimum, and difference between the maximum and minimum features. Next, the method constructs a feature vector concatenating the features and recognizes the vector sequence by using HMMs with grammars.

- $R F+H M M$ : This method is a combination of $R F$ and $H M M$. This method recognizes the time series of output class probabilities of $R F$ by using HMMs with grammars.

- $C N N$ : This method directly processes the raw amplitude and phase data in addition to the amplitude and phase information extracted from the decomposed CSI within a window by using the convolutional neural networks shown in Fig. 6 and classifies the window into an appropriate class. We added a densely connected layer with 128 nodes and a softmax output layer below the last layers in Fig. 6 . That is, the output of the four convolutional neural networks is fed into the densely connected layer, and then the output of the densely connected layer is fed into the softmax output layer. (We prepare convolutional layers for four inputs: raw amplitude, raw phase, decomposed amplitude, and decomposed phase.)

Recognition accuracy for each of the above methods is evaluated using the macro-averaged precision, recall, and F-measure, calculated based on the recognition results per window of data. Note that, because we prepare a classifier for each object, we compute the average precision, recall, and F-measure for each object in each environment. Experimental parameters used in this study are shown in Table 3 and were determined based on a preliminary experiment. 
Detecting State Changes of Indoor Everyday Objects using Wi-Fi Channel State Information • 88:17

Table 10. Classification accuracies for Proposed related to door in environment 1

\begin{tabular}{|c||c|c|c|}
\hline & Precision & Recall & F-measure \\
\hline \hline open & 0.670 & 0.835 & 0.743 \\
\hline close & 0.676 & 0.901 & 0.773 \\
\hline opened & 0.990 & 0.976 & 0.983 \\
\hline closed & 0.991 & 0.973 & 0.982 \\
\hline
\end{tabular}

Table 12. Classification accuracies for Proposed related to window 2 in environment 1

\begin{tabular}{|c||c|c|c|}
\hline & Precision & Recall & F-measure \\
\hline \hline open & 0.617 & 0.831 & 0.708 \\
\hline close & 0.666 & 0.990 & 0.796 \\
\hline opened & 0.985 & 0.962 & 0.973 \\
\hline closed & 0.996 & 0.973 & 0.984 \\
\hline
\end{tabular}

Table 11. Classification accuracies for Proposed related to window 1 in environment 1

\begin{tabular}{|c||c|c|c|}
\hline & Precision & Recall & F-measure \\
\hline \hline open & 0.638 & 0.868 & 0.735 \\
\hline close & 0.706 & 0.908 & 0.795 \\
\hline opened & 0.956 & 0.972 & 0.964 \\
\hline closed & 0.997 & 0.973 & 0.985 \\
\hline
\end{tabular}

Table 13. Classification accuracies for Proposed related to shade 1 in environment 1

\begin{tabular}{|c||c|c|c|}
\hline & Precision & Recall & F-measure \\
\hline \hline open & 0.750 & 0.877 & 0.809 \\
\hline close & 0.806 & 0.941 & 0.868 \\
\hline opened & 0.929 & 0.973 & 0.950 \\
\hline closed & 0.984 & 0.893 & 0.936 \\
\hline
\end{tabular}

\subsection{Results}

4.3.1 Classification accuracy for the proposed method. Tables 7, 8, and 9 show the classification accuracies for Proposed in the three environments. As shown in the results, our method achieved an accuracy of about $85 \%$ in the three environments. Tables 10-15 show the classification accuracies for the six objects in environment 1 , and the accuracies for the "open" and "close" events were somewhat poor. In contrast, the accuracies for the "opened" and "closed" states for these objects were near-perfect. The upper portion of Fig. 9 shows an example of the outputs of our method related to "door" in environment 1. As the results show, the estimated start times and end times of the "open" and "close" events have small errors. Because the number of instances belonging to the "open" and "close" classes is small, the small errors greatly affected the classification accuracies for these classes. However, as shown in the upper portion of Fig. 9, our method could capture the state changes of objects, and the accuracies for the "opened" and "closed" states are very high. We believe that many of the applications based on object use such as adaptive HVAC control and monitoring an independently living elderly person can work well with only information about the state changes of objects.

Figure 10 shows visual confusion matrices for Proposed in environment 1. The classification accuracies for "screen" in environment 1 were somewhat poor. As shown in Fig. 8, the screen is located close to the wall. Therefore, we believe that, even when the screen was pulled down, a signal reflection path did not change significantly. In addition, the accuracies for "window shade 1" and "window 1" seem to be somewhat higher than those for "window shade 2" and "window 2." This is because "window shade 1" and "window 1" are located close to the Wi-Fi AP, as shown in Fig. 7. Similarly, the accuracies for "door" in environment 2 and "window" and "shade" in environment 3, which are also located closed to the AP or PC, were high, as shown in Tables 8 and 9.

As can be seen in Table 8, the accuracies for "shade" in environment 2 were somewhat poor. In addition, the accuracies for "shade 2 " in environment 1 were somewhat poor. This may be because the distance between a window and the window shade associated with the window is very short. We believe that we can cope with this problem by incorporating grammars describing the relationships between two adjacent correlated objects. For example, when the door is in the "opened" state, the shade should also be in the "opened" state. Incorporating such grammars in the HMM decoding is one of our important future tasks.

In environments 2 and 3, our method could successfully recognize the events of the three adjacent cabinets as shown in Tables 8 and 9. In contrast, the accuracies for "refrigerator" in environment 3 were poor. Because 
$88: 18 \cdot$ K. Ohara et al.

Table 14. Classification accuracies for Proposed related to shade 2 in environment 1

\begin{tabular}{|c||c|c|c|}
\hline & Precision & Recall & F-measure \\
\hline \hline open & 0.770 & 0.774 & 0.772 \\
\hline close & 0.672 & 0.985 & 0.799 \\
\hline opened & 0.862 & 0.979 & 0.916 \\
\hline closed & 0.992 & 0.742 & 0.849 \\
\hline
\end{tabular}

Table 15. Classification accuracies for Proposed related to screen in environment 1

\begin{tabular}{|c||c|c|c|}
\hline & Precision & Recall & F-measure \\
\hline \hline pull-down & 0.599 & 0.877 & 0.712 \\
\hline retract & 0.621 & 0.783 & 0.692 \\
\hline pulled-down & 0.962 & 0.971 & 0.967 \\
\hline retracted & 0.981 & 0.937 & 0.959 \\
\hline
\end{tabular}
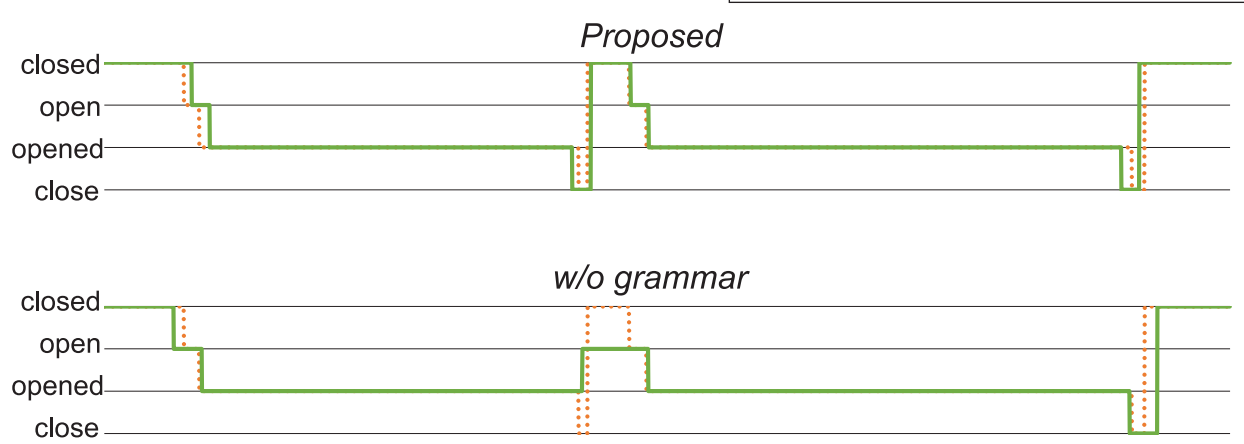

close

w/o HMM

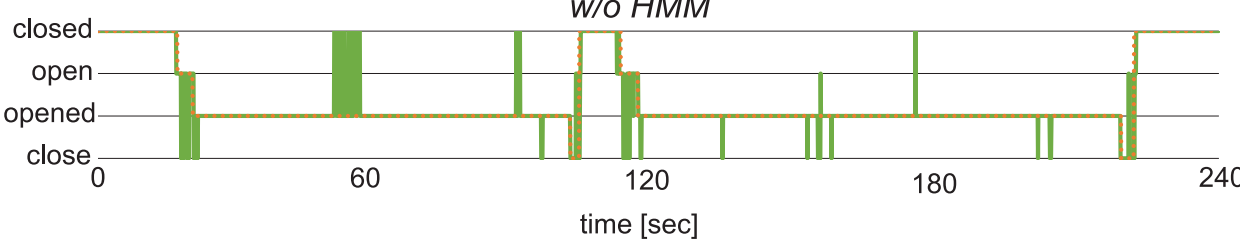

Fig. 9. Outputs of Proposed, w/o grammar, and w/o HMM for door in environment 1 over a continuous 240-s segment of the data. The graph also shows the ground truth, which was obtained by annotating video recorded from a web camera.

the refrigerator used in the environment was small, when a participant used the refrigerator, it was occluded by the participant. Therefore, sensor data collected when the refrigerator was used were similar to the sensor data collected when the participant walked in front of the refrigerator.

4.3.2 Contribution of ICA. Figure 11 shows the F-measures of the nine methods in the three environments. As shown in the results, when we do not use ICA, the F-measures decrease by about 1\%-5\%. ICA improves the accuracies for only the several objects that are located close to other objects such as windows and cabinets. This may be because ICA can extract independent components that enable us to distinguish the confusing events of these objects.

Tables 16, 17, and 18 compare the recognition accuracies of Proposed and those of w/o ICA. As shown in the tables, substantial performance improvements are achieved for objects whose recognition accuracies in $w / o$ $I C A$ were poor. Accuracy of $w / o I C A$ and performance improvement have a strong negative correlation, with a correlation coefficient of -0.755 . Although the accuracies for objects located close to other objects are inherently

Proceedings of the ACM on Interactive, Mobile, Wearable and Ubiquitous Technologies, Vol. 1, No. 3, Article 88. Publication date: September 2017. 
Detecting State Changes of Indoor Everyday Objects using Wi-Fi Channel State Information • 88:19
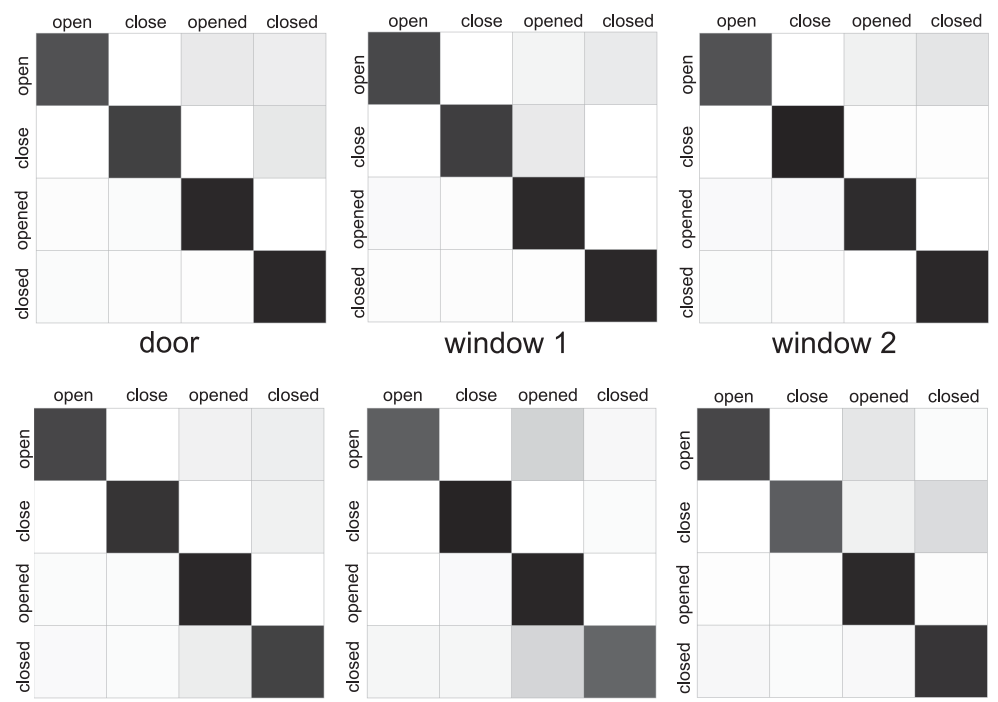

shade 2

window 2

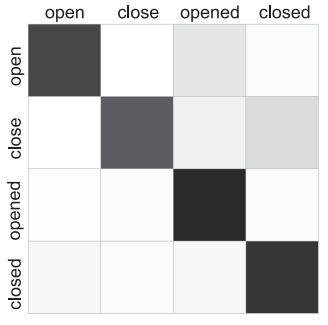

screen

Fig. 10. Visual confusion matrices for Proposed in environment 1

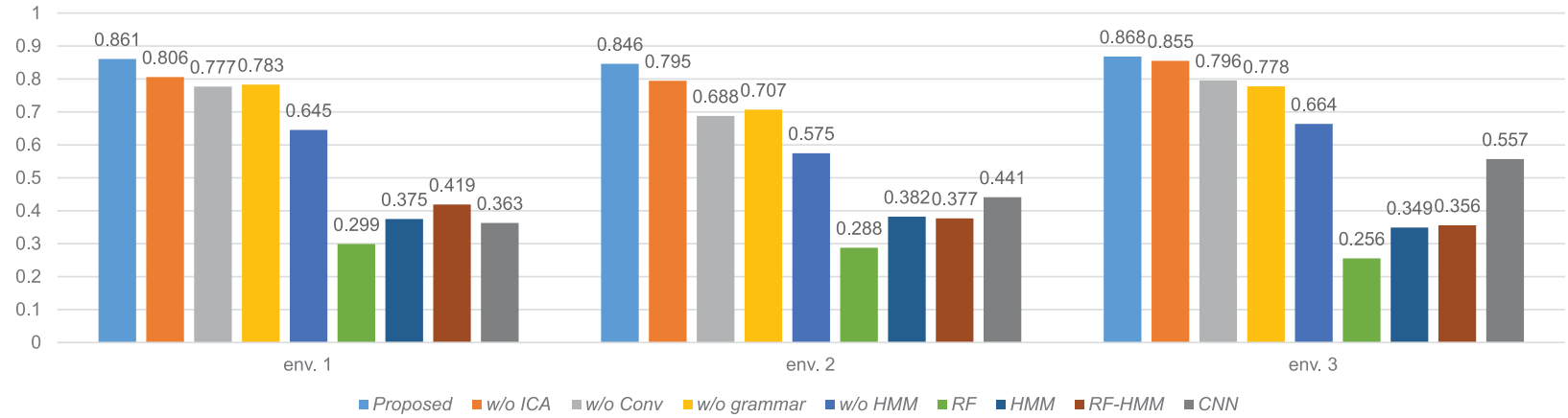

Fig. 11. F-measures of the methods in the three environments

poor in our method, ICA can extract independent components that enable us to distinguish the confusing events of these objects. In fact, the accuracies for doors did not improve even when ICA was used because there were no objects around the doors. In contrast, the accuracies for many windows, shades, and cabinets improved. The improvement for the "projector screen" was large because the events/states of this object do not change the signal propagation paths considerably, as aforementioned. Therefore, the changes in the propagation paths caused by "projector screen" are obscured by the changes in the propagation paths caused by other objects, and ICA can deal well with such situations. As mentioned above, we could confirm that ICA is effective for objects whose events/states are confused with those of other objects.

4.3.3 Contributions of grammar and HMMs. As shown in Fig. 11, when we do not use the handcrafted grammars, the F-measures decrease by about $8 \%-15 \%$. Therefore, the contribution of the handcrafted grammars is somewhat higher than that of ICA. The grammars evenly improve the accuracies for all objects. In addition, when 
Table 16. Comparison of F-measures for Proposed and $w / 0$ ICA in environment 1

\begin{tabular}{|c||c|c|c|}
\hline Object & Proposed & w/o ICA & Improvement \\
\hline \hline door & 0.870 & 0.856 & 0.014 \\
\hline window 1 & 0.870 & 0.795 & 0.075 \\
\hline window 2 & 0.866 & 0.840 & 0.026 \\
\hline shade 1 & 0.891 & 0.881 & 0.010 \\
\hline shade 2 & 0.834 & 0.777 & 0.057 \\
\hline screen & 0.832 & 0.685 & 0.147 \\
\hline
\end{tabular}

Table 17. Comparison of F-measures for Proposed and $w / o$ ICA in environment 2

\begin{tabular}{|c||c|c|c|}
\hline Object & Proposed & w/o ICA & Improvement \\
\hline \hline door & 0.877 & 0.853 & 0.024 \\
\hline window & 0.849 & 0.771 & 0.078 \\
\hline shade & 0.804 & 0.820 & -0.016 \\
\hline cabinet 1 & 0.849 & 0.720 & 0.129 \\
\hline cabinet 2 & 0.855 & 0.819 & 0.036 \\
\hline cabinet 3 & 0.844 & 0.784 & 0.060 \\
\hline
\end{tabular}

Table 18. Comparison of F-measures for Proposed and w/o ICA in environment 3

\begin{tabular}{|c||c|c|c|}
\hline Object & Proposed & w/o ICA & Improvement \\
\hline \hline window & 0.888 & 0.872 & 0.016 \\
\hline shade & 0.924 & 0.930 & -0.006 \\
\hline cabinet 1 & 0.846 & 0.839 & 0.007 \\
\hline cabinet 2 & 0.879 & 0.870 & 0.009 \\
\hline cabinet 3 & 0.877 & 0.852 & 0.025 \\
\hline refrigerator & 0.795 & 0.767 & 0.028 \\
\hline
\end{tabular}

we do not use HMMs, the F-measures greatly decrease by about 10\%-20\%, and we can say that the HMMs (with grammars) are the best contributor. When we did not use the HMMs, many sporadic errors (false detections of events) were observed. These errors were caused when the participant walked around the objects or other adjacent objects were used.

Figure 9 also shows example outputs of w/o grammar and $w / o$ HMM for the door in environment 1 . As shown in the figure, when we do not use grammar, the method outputs impossible state/event transitions, for example, "open" event to "opened" state. In addition, we can find many sporadic errors in $w / o$ HMM. This may be because the participant walked around the door and AP to use other objects. As above, the HMMs are essential for smoothing out sporadic errors caused by noisy CSI data.

4.3.4 Contribution of feature learning. As shown in Fig. 11, when we do not use the convolutional layers for feature learning, the F-measures decrease by about $7 \%-15 \%$. We can say that the contribution of the convolutional layers is high and almost the same as that of the grammars. The convolutional layers also evenly improve the accuracies for all the objects.

Because the DNN learns features to maximize the recognition accuracy, each neuron (node) in the network is said to be an event/state detector. Here to intuitively show what the DNN learns, we show the outputs of the DNN for "door" in environment 1, that is, time-series of activations of nodes of the last LSTM layer, in Fig. 12. Because the last layer has 128 nodes, the time-series is 128-dimensional data. As shown in the figure, several nodes are trained as detectors for only open and close events ((a) in Fig. 12) and do not distinguish between open and close events. In contrast, many other nodes can distinguish between the opened state and the closed state ((b) in Fig. 12). In addition, several nodes ignore close events ((c) in Fig. 12). As aforementioned, we can learn event/state detectors with different properties by using the DNN. Fig. 13 shows the time-series of activations for "projector screen." Interestingly, although the DNN is tailored to the object, the activations are affected by the events of other objects. This means that it was difficult for the DNN to find good feature representations to detect events/states of "projector screen." As aforementioned, the events/states of this object do not change the signal propagation paths considerably. 
Detecting State Changes of Indoor Everyday Objects using Wi-Fi Channel State Information • 88:21

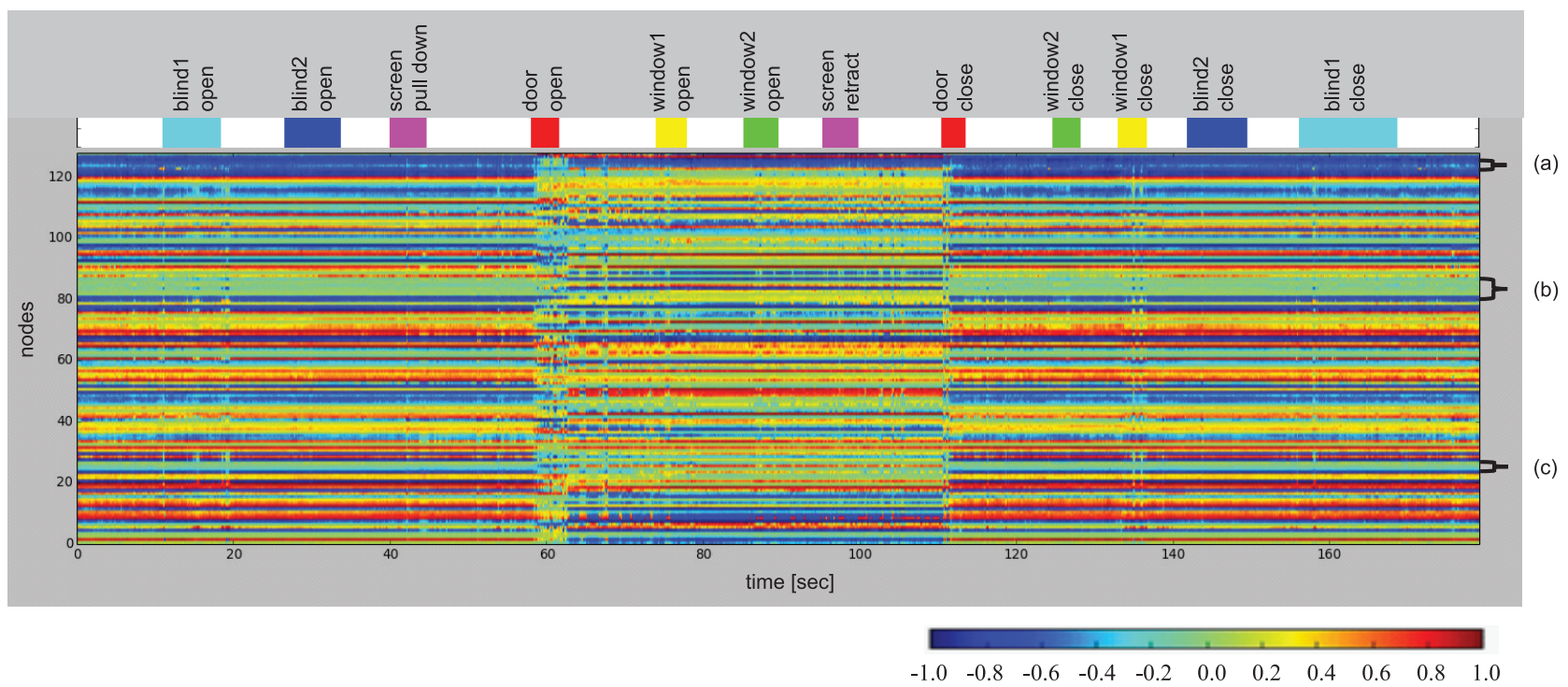

Fig. 12. Time-series of activations of nodes of the last LSTM layer for door in environment 1

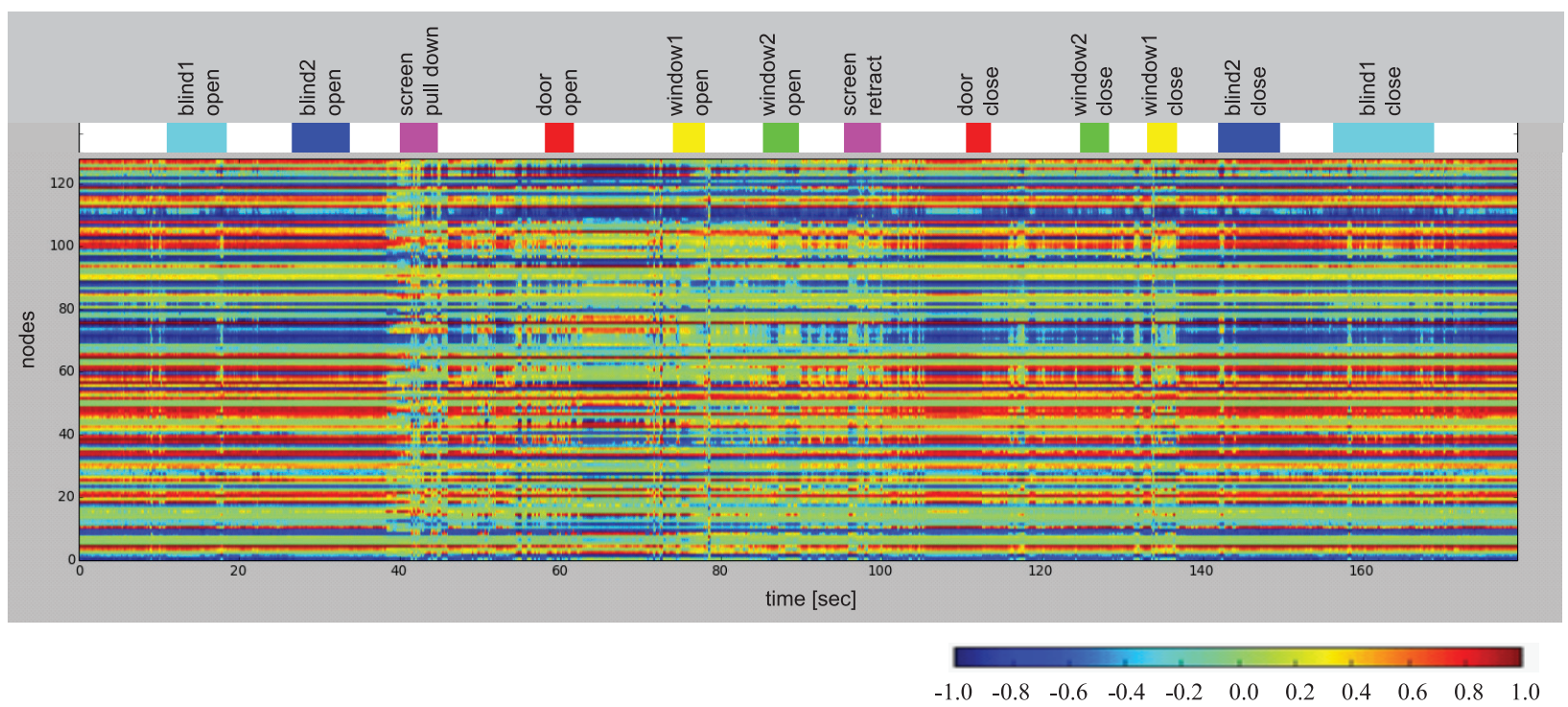

Fig. 13. Time-series of activations of nodes of the last LSTM layer for screen in environment 1

In addition, as shown in Fig. 11, the accuracies for $R F$ are much poorer than those for Proposed even though $R F$ employs a promising approach, i.e., a combination of PCA and random forests, used in recent state-of-the-art studies. These results indicate the limitation of the handcrafted features in this task. As above, we could confirm the effectiveness of our feature learning-based approach. 
$88: 22 \cdot$ K. Ohara et al.

Table 19. Effect of usage of door (used from inside/outside) on recognition accuracies

\begin{tabular}{|c|c||c|c|c|}
\hline Train & Test & Precision & Recall & F-measure \\
\hline \hline inside & inside & 0.812 & 0.910 & 0.838 \\
\hline inside & outside & 0.833 & 0.886 & 0.843 \\
\hline outside & inside & 0.664 & 0.794 & 0.678 \\
\hline outside & outside & 0.913 & 0.937 & 0.923 \\
\hline two-side & inside & 0.769 & 0.862 & 0.785 \\
\hline two-side & outside & 0.785 & 0.838 & 0.797 \\
\hline two-side (180) & inside & 0.804 & 0.914 & 0.835 \\
\hline two-side (180) & outside & 0.861 & 0.927 & 0.877 \\
\hline
\end{tabular}

Moreover, as shown in the results of $R F+H M M$, even when we incorporate the HMMs (with grammar), which is the best contributor in our method, the accuracies do not improve greatly. Fig. 11 also shows the results of $C N N$, and $C N N$ outperforms $R F$. These results also indicate the usefulness of feature learning. However, the accuracies of $C N N$ are poorer than those of our method. From the above results, we could confirm the usefulness of the blind source separation, feature learning, and HMMs.

\subsection{Discussion}

4.4.1 Effects of additional persons and objects. The data used in the above experiments were collected when there were no other persons in the environments. To investigate the effects of another person in the same room, we collected an additional 10 sessions of data in environment 3 when there was another person walking at random in the environment. We recognize the data using the network trained on data collected for one person, and the average F-measure was 0.821. Because the person changed the signal propagation paths, the accuracy decreased by about $4 \%$. However, the F-measure related to states, i.e., "opened" and "closed," is still high and is about 0.917 .

Here, we also investigate the effects of additional objects that can change the signal propagation paths. We installed four additional chairs in environment 3 and collected 10 sessions of test data. We recognized the data using a network trained on the data collected before the object additions, and the average F-measure was 0.782 . The four new chairs drastically changed the signal propagation paths and the accuracy decreased by about $8 \%$. However, the F-measure related to states remains high and is about 0.871 , even though we drastically changed the environment.

4.4.2 Effect of usage of objects. Here we investigate the effects of the usage of an object on the recognition accuracy. We prepared our recognition model for "door" in environment 1 that was trained on labeled data collected when the door was used from the inside of the room (inside model). We tested the model by using data collected when the door was used from the inside or outside of the room. We also prepared a model trained using data collected when the door was used from the inside of the room (outside model) and tested the model by using data collected when the door was used from the inside or outside. In addition, we prepared a model trained on data including "open" and "close" from both inside and outside of the room (two-side model). Note that the durations of training data for these three models are almost identical. The number of training "open/close" events is 90 . The number of test "open/close" events is 10.)

Table 19 shows the results of our method. Interestingly, the accuracies of the inside model model are fine for both the inside and the outside test data. In contrast, the accuracies of the outside model are poor for the inside test data. When the participant used the door from the inside, CSI data included noises caused by the participant because the AP was inside the room. However, when the participant used the door from the outside, Wi-Fi signals were not affected by the body of the participant. Thus, the CSI data are thought to be "clean." We believe that

Proceedings of the ACM on Interactive, Mobile, Wearable and Ubiquitous Technologies, Vol. 1, No. 3, Article 88. Publication date: September 2017. 


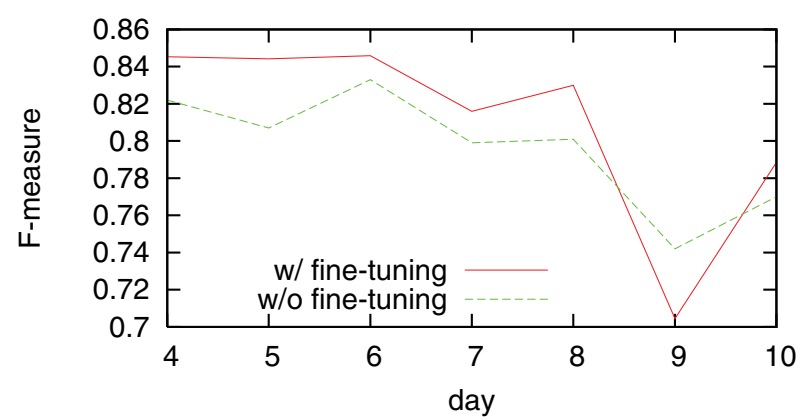

Fig. 14. Transitions of F-measures when we do or do not perform fine-tuning

the inside model, which is trained using noisy data, is robust and can deal well with the outside test data. We also found that the accuracies of the two-side model are poorer than those of the inside model. This may be because the training data used for the two-side model included only 45 "open/close" events (90 events in total). When we doubled the amount of training data (two-side (180) rows in Table 19), the accuracies improved by approximately $5-8 \%$.

4.4.3 Evaluation with long-term data. We collected sensor data for ten days in environment 2, where 20 sessions of data were collected per day, to investigate the stability of the performance of our method. We trained the classifier on the first three days of data and recognized the remaining data using the trained classifier. Note that, during the data collection period, the positions of objects such as chairs could slightly change and the temperature and humidity also could change. Therefore, the signal condition in the environment gradually changed over time. To cope with this problem, we employed an adaptation technique to adapt the neural network to the environmental dynamics by updating the network every day using unlabeled test data collected on that day. After we obtained the test data, we fine-tuned the network simply by recognizing the test data with the network and then re-training (updating) the parameters of the network using the recognized results as pseudo-ground truth. Note that the fine-tuning is not performed in the above evaluations.

Figure 14 shows the transitions of the F-measures, and we can confirm that using the fine-tuning improved the accuracy by about 2\%. As for the first three days (days 4-6 in Fig. 14), we could achieve good accuracies about 0.845 even though the network was trained on a small amount of training data. However, the F-measure suddenly decreased on day 9. This may be because day 9 (and day 10) was rainy. In contrast, all the other days were sunny or cloudy. Because the humidity greatly affects the radio transmission, weather-aware network tuning may be useful. Fig. 15 shows the relationship between the classification accuracy and the environmental conditions (city-level humidity and temperature). As shown in the figure, when humidity increased suddenly on the 9th day, accuracy decreased suddenly. In contrast, temperature did not seem to affect accuracy. However, humidity and accuracy do not have a strong correlation, with a correlation coefficient of -0.360 . From the above, we cannot say that humidity is correlated directly to classification accuracy. However, the sudden changes in humidity due to rain do affect recognition accuracy. As a part of our future work, we plan to investigate the relationship between recognition accuracy and room-level humidity.

Figure 16 shows the transitions of the F-measures for states and events ("states w/ 3-day train" and "events w/ 3 -day train" in the graph). Even on rainy days, i.e., the days 9 and 10, our method maintains good performance in terms of state recognition. In contrast, the F-measure for events greatly decreases on day 9 . We conclude that state recognition using our method is robust to external factors such as weather and the movements of objects. As mentioned above, many of the applications based on object use can be implemented solely using estimated states. 
$88: 24 \cdot$ K. Ohara et al.
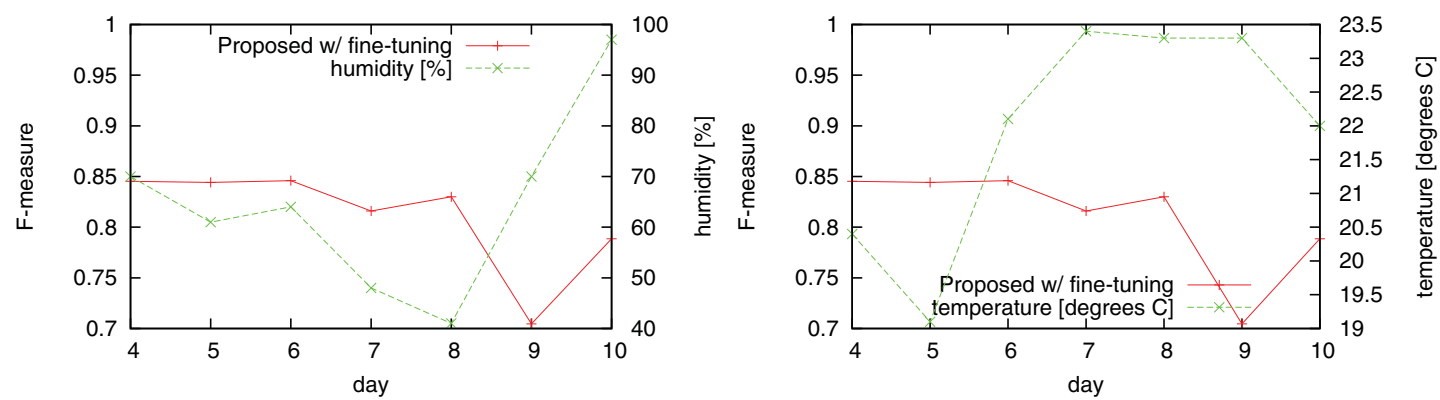

Fig. 15. Effects of humidity and temperature on recognition accuracies

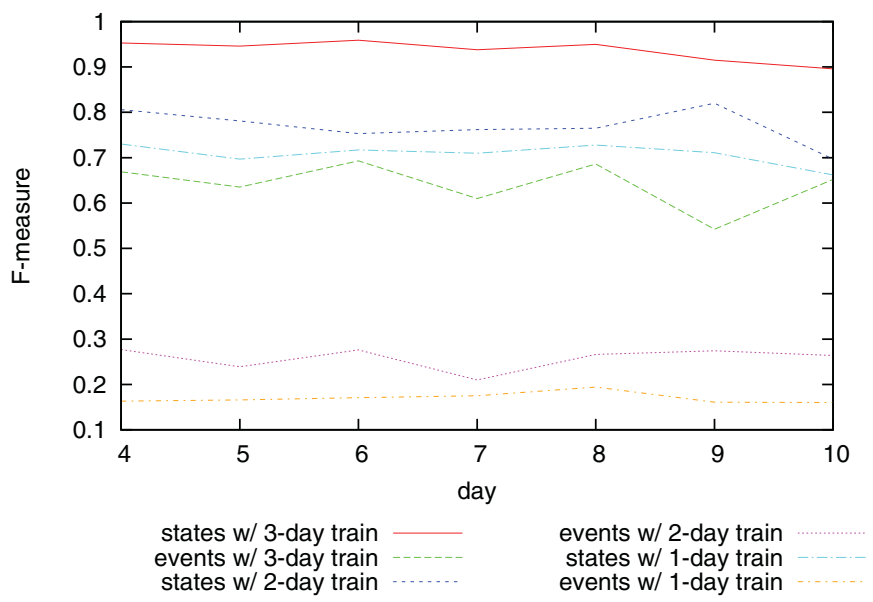

Fig. 16. Transitions of F-measures for events and states when the amount of training data is varied

4.4.4 Amount of training data. In the above investigation, we used only the first three days of data as training sessions and confirmed that our method achieved high recognition accuracies using this amount of data. Here, we investigate the effect of the amount of the training data by reducing the amount, and Fig. 17 shows the change in F-measures in environment 2 when we use the first day, two days, or three days of data as training data. As shown in the figure, when we use only two days or one day of training data, the F-measures decrease by about 0.3-0.4 compared with the F-measures when three days of training data are used.

Fig. 16 shows the transitions of the F-measures for states and events when we use the first day, two days, or three days of data as training data. As shown in the figure, the F-measures for the states when two days of training data are used are relatively good and are about 0.80 . In contrast, the F-measures for events significantly decrease when two days or one day of training data are used. This may be because the amount of training data for events is much smaller than that for states, as events such as "open" and "close" last for a short time.

Here, the three days of training data correspond to about 120 min of data in our experiment, and collecting this amount of data is laborious because we must open and close doors and windows many times for two hours. However, collecting $120 \mathrm{~min}$ of data for only states is relatively easier. We can collect training data while going out, sleeping, preparing meals, or watching television by keeping a door opened or closed.

Proceedings of the ACM on Interactive, Mobile, Wearable and Ubiquitous Technologies, Vol. 1, No. 3, Article 88. Publication date: September 2017. 
Detecting State Changes of Indoor Everyday Objects using Wi-Fi Channel State Information • 88:2

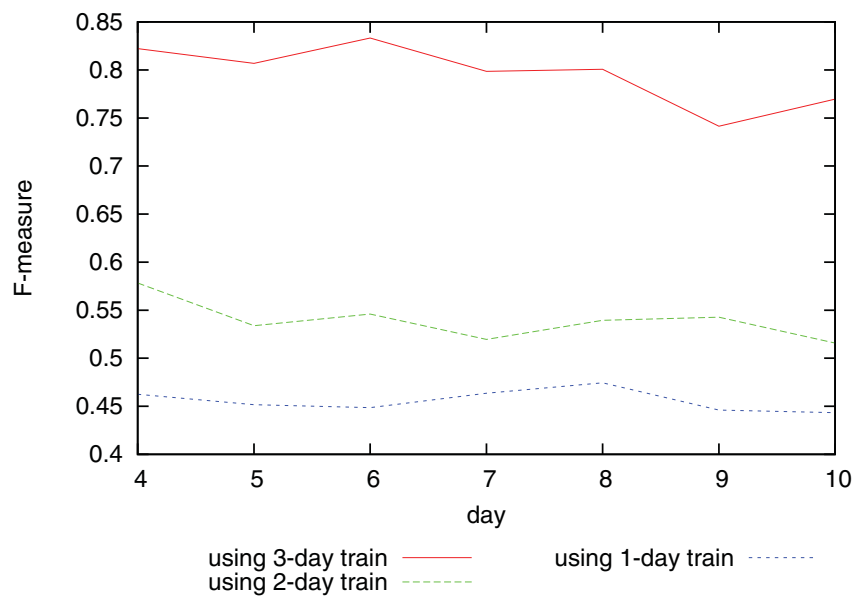

Fig. 17. Transitions of F-measures when the amount of training data is varied

Table 20. Recognition accuracies of our method with/without additional AP

\begin{tabular}{|c||c|c|c|}
\hline Additional AP & Precision & Recall & F-measure \\
\hline \hline yes & 0.802 & 0.891 & 0.823 \\
\hline no & 0.846 & 0.920 & 0.870 \\
\hline
\end{tabular}

To further reduce the amount of labeled training data, we can apply semi-supervised learning and/or active learning $[9,13]$. Semi-supervised learning is effective in an environment where unlabeled data are sufficient. Because we assume home environments, we can obtain data $24 \mathrm{~h}$ per day. In active learning, the learning algorithm interactively queries a user to obtain the ground truth of a data point. In our case, when sensor data that are difficult to interpret are observed, the algorithm asks the user to input the ground truth, that is, the event or the state of an object. We believe that the active learning technique can be applied effectively with unsupervised segmentation techniques such as [22]. Because the states of indoor objects do not change frequently, the segmentation algorithm can output a sensor data segment corresponding to one state and the duration of the segment can be few minutes to several hours. By labeling the segment, we can easily collect long-duration labeled sensor data.

4.4.5 Effect of interference. To investigate the effect of interference from another AP, we collected additional data for the door in environment 1 when we placed another AP at a distance of $1 \mathrm{~m}$ from our AP. We trained our recognition model by using training data containing 90 open/close events (180 in total) and tested the model by using test data containing 10 open/close events. In addition, we collected data for the door when the additional AP was removed and trained and tested our model by using this data. Table 20 shows the recognition accuracies. As shown in the results, when we placed the additional AP, the recognition accuracy decreased approximately by $5 \%$. From the above, we can confirm the effect of a nearby AP, and we can say that such wireless devices should not be placed near an AP for data collection.

\section{CONCLUSION}

This study proposed a method for detecting events and states of indoor everyday objects such as doors and windows using Wi-Fi CSI obtained from a commercial Wi-Fi module. We designed a novel processing pipeline based 
on blind source separation, deep learning, and hidden Markov models based on a grammar that describes knowledge about the state transitions of an object. We then investigated the effectiveness of the classifier using real data. Our evaluation experiment revealed that our approach significantly outperformed a classic machine learning-based approach. However, because our method relies on deep learning, scant training data results in poor recognition accuracy, although it still substantially outperformed the classic approach. Coping with this problem is one of our important future tasks.

\section{REFERENCES}

[1] Heba Abdel-Nasser, Reham Samir, Ibrahim Sabek, and Moustafa Youssef. 2013. MonoPHY: Mono-stream-based device-free WLAN localization via physical layer information. In IEEE Wireless Communications and Networking Conference (WCNC 2013). 4546-4551.

[2] Heba Abdelnasser, Khaled A Harras, and Moustafa Youssef. 2015. UbiBreathe: A ubiquitous non-invasive WiFi-based breathing estimator. In the 16th ACM International Symposium on Mobile Ad Hoc Networking and Computing. 277-286.

[3] Fadel Adib, Zach Kabelac, Dina Katabi, and Robert C Miller. 2014. 3D tracking via body radio reflections. In the 11th USENIX Symposium on Networked Systems Design and Implementation (NSDI 14). 317-329.

[4] Kamran Ali, Alex Xiao Liu, Wei Wang, and Muhammad Shahzad. 2015. Keystroke recognition using WiFi signals. In the 21st Annual International Conference on Mobile Computing and Networking (MobiCom 2015). 90-102.

[5] Chris Beckmann, Sunny Consolvo, and Anthony LaMarca. 2004. Some assembly required: Supporting end-user sensor installation in domestic ubiquitous computing environments. In International Conference on Ubiquitous Computing (UbiComp 2004). 107-124.

[6] Yoshua Bengio, Patrice Simard, and Paolo Frasconi. 1994. Learning long-term dependencies with gradient descent is difficult. IEEE Transactions on Neural Networks 5, 2 (1994), 157-166.

[7] Leo Breiman. 2001. Random forests. Machine learning 45, 1 (2001), 5-32.

[8] Bradford Campbell and Prabal Dutta. 2014. An energy-harvesting sensor architecture and toolkit for building monitoring and event detection. In the 1st ACM Conference on Embedded Systems for Energy-Efficient Buildings. 100-109.

[9] Olivier Chapelle, Bernhard Schölkopf, and Alexander Zien. Semi-supervised learning. MIT press Cambridge, MA.

[10] Yogita Chapre, Aleksandar Ignjatovic, Aruna Seneviratne, and Sanjay Jha. 2015. CSI-MIMO: An efficient Wi-Fi fingerprinting using Channel State Information with MIMO. Pervasive and Mobile Computing 23 (2015), 89-103.

[11] Ricardo Chavarriaga, Hesam Sagha, Alberto Calatroni, Sundara Tejaswi Digumarti, Gerhard Tröster, José del R Millán, and Daniel Roggen. 2013. The Opportunity challenge: A benchmark database for on-body sensor-based activity recognition. Pattern Recognition Letters 34, 15 (2013), 2033-2042.

[12] Dan Ciregan, Ueli Meier, and Jürgen Schmidhuber. 2012. Multi-column deep neural networks for image classification. In Computer Vision and Pattern Recognition (CVPR 2012). 3642-3649.

[13] David Cohn, Les Atlas, and Richard Ladner. 1994. Improving generalization with active learning. Machine Learning 15, 2 (1994), 201-221.

[14] Pierre Comon. 1994. Independent component analysis, a new concept? Signal Processing 36, 3 (1994), $287-314$.

[15] Felix A Gers, Jürgen Schmidhuber, and Fred Cummins. 2000. Learning to forget: Continual prediction with LSTM. Neural Computation 12,10 (2000), 2451-2471.

[16] Alex Graves, Abdel-rahman Mohamed, and Geoffrey Hinton. 2013. Speech recognition with deep recurrent neural networks. In the 38th International Conference on Acoustics, Speech and Signal Processing (ICASSP 2013). 6645-6649.

[17] Yanying Gu, Anthony Lo, and Ignas Niemegeers. 2009. A survey of indoor positioning systems for wireless personal networks. IEEE Communications Surveys \& Tutorials 11, 1 (2009), 13-32.

[18] Daniel Halperin, Wenjun Hu, Anmol Sheth, and David Wetherall. 2011. Tool release: Gathering $802.11 \mathrm{n}$ traces with channel state information. ACM SIGCOMM Computer Communication Review 41, 1 (2011), 53-53.

[19] Chunmei Han, Kaishun Wu, Yuxi Wang, and Lionel M Ni. 2014. WiFall: Device-free fall detection by wireless networks. In IEEE Conference on Computer Communications (INFOCOM 2014). 271-279.

[20] Aapo Hyvärinen. 1999. Fast and robust fixed-point algorithms for independent component analysis. IEEE Transactions on Neural Networks 10, 3 (1999), 626-634.

[21] Sergey Ioffe and Christian Szegedy. 2015. Batch normalization: Accelerating deep network training by reducing internal covariate shift. arXiv preprint arXiv:1502.03167 (2015).

[22] Matthew J. Johnson and Alan S. Willsky. 2013. Bayesian nonparametric hidden semi-Markov models. Journal of Machine Learning Research 14, 1 (2013), 673-701.

[23] Diederik Kingma and Jimmy Ba. 2014. Adam: A method for stochastic optimization. arXiv preprint arXiv:1412.6980 (2014).

[24] Palanivel A Kodeswaran, Ravi Kokku, Sayandeep Sen, and Mudhakar Srivatsa. 2016. Idea: A system for efficient failure management in smart IoT environments. In the 14th Annual International Conference on Mobile Systems, Applications, and Services (MobiSys 2016).

Proceedings of the ACM on Interactive, Mobile, Wearable and Ubiquitous Technologies, Vol. 1, No. 3, Article 88. Publication date: September 2017. 
$43-56$.

[25] Anthony LaMarca, Yatin Chawathe, Sunny Consolvo, Jeffrey Hightower, Ian Smith, James Scott, Timothy Sohn, James Howard, Jeff Hughes, Fred Potter, and others. 2005. Place lab: Device positioning using radio beacons in the wild. In International Conference on Pervasive Computing (Pervasive 2005). 116-133.

[26] Hui Liu, Houshang Darabi, Pat Banerjee, and Jing Liu. 2007. Survey of wireless indoor positioning techniques and systems. IEEE Transactions on Systems, Man, and Cybernetics, Part C: Applications and Reviews 37, 6 (2007), 1067-1080.

[27] Xuefeng Liu, Jiannong Cao, Shaojie Tang, and Jiaqi Wen. 2014. Wi-Sleep: Contactless sleep monitoring via WiFi signals. In IEEE Real-Time Systems Symposium (RTSS 2014). 346-355.

[28] Kazuya Ohara, Takuya Maekawa, Yasue Kishino, Yoshinari Shirai, and Futoshi Naya. 2015. Transferring positioning model for devicefree passive indoor localization. In the 2015 ACM International Joint Conference on Pervasive and Ubiquitous Computing (UbiComp 2015). 885-896.

[29] Francisco Javier Ordóñez and Daniel Roggen. 2016. Deep convolutional and LSTM recurrent neural networks for multimodal wearable activity recognition. Sensors 16, 1 (2016), 115.

[30] Shwetak N Patel, Matthew S Reynolds, and Gregory D Abowd. 2008. Detecting human movement by differential air pressure sensing in HVAC system ductwork: An exploration in infrastructure mediated sensing. In International Conference on Pervasive Computing (Pervasive 2008). 1-18.

[31] Anindya S Paul, Eric A Wan, Fatema Adenwala, Erich Schafermeyer, Nick Preiser, Jeffrey Kaye, and Peter G Jacobs. 2014. MobileRF: A robust device-free tracking system based on a hybrid neural network HMM classifier. In the 2014 ACM International Joint Conference on Pervasive and Ubiquitous Computing (UbiComp 2014). 159-170.

[32] Matthai Philipose, Kenneth P Fishkin, Mike Perkowitz, Donald J Patterson, Dieter Fox, Henry Kautz, and Dirk Hähnel. 2004. Inferring activities from interactions with objects. IEEE Pervasive Computing 3, 4 (2004), 50-57.

[33] Qifan Pu, Sidhant Gupta, Shyamnath Gollakota, and Shwetak Patel. 2013. Whole-home gesture recognition using wireless signals. In the 19th annual International Conference on Mobile Computing and Networking (MobiCom 2013). 27-38.

[34] Lawrence R Rabiner. 1989. A tutorial on hidden Markov models and selected applications in speech recognition. Proc. IEEE 77, 2 (1989), 257-286.

[35] Moustafa Seifeldin, Ahmed Saeed, Ahmed E Kosba, Amr El-Keyi, and Moustafa Youssef. 2012. Nuzzer: A large-scale device-free passive localization system for wireless environments. IEEE Transactions on Mobile Computing 12, 7 (2012), 1321-1334.

[36] Souvik Sen, Božidar Radunovic, Romit Roy Choudhury, and Tom Minka. 2012. You are facing the Mona Lisa: Spot localization using PHY layer information. In the 10th International Conference on Mobile Systems, Applications, and Services. 183-196.

[37] Shuyu Shi, Stephan Sigg, and Yusheng Ji. 2012. Passive detection of situations from ambient FM-radio signals. In the 2012 ACM Conference on Ubiquitous Computing (UbiComp 2012). 1049-1053.

[38] Stephan Sigg, Ulf Blanke, and Gerhard Tröster. 2014. The telepathic phone: Frictionless activity recognition from WiFi-RSSI. In 2014 IEEE International Conference on Pervasive Computing and Communications (PerCom 2014). 148-155.

[39] Nitish Srivastava, Geoffrey E Hinton, Alex Krizhevsky, Ilya Sutskever, and Ruslan Salakhutdinov. 2014. Dropout: A simple way to prevent neural networks from overfitting. Journal of Machine Learning Research 15, 1 (2014), 1929-1958.

[40] Emmanuel Munguia Tapia, Stephen S Intille, and Kent Larson. 2004. Activity recognition in the home using simple and ubiquitous sensors. In International Conference on Pervasive Computing (Pervasive 2004). 158-175.

[41] Tim Van Kasteren, Athanasios Noulas, Gwenn Englebienne, and Ben Kröse. 2008. Accurate activity recognition in a home setting. In the 10th International Conference on Ubiquitous Computing (Ubicomp 2008). 1-9.

[42] Wei Wang, Alex X Liu, Muhammad Shahzad, Kang Ling, and Sanglu Lu. 2015. Understanding and modeling of WiFi signal based human activity recognition. In the 21st annual International Conference on Mobile Computing and Networking (MobiCom 2015). 6576.

[43] Xuyu Wang, Lingjun Gao, and Shiwen Mao. 2015. PhaseFi: Phase fingerprinting for indoor localization with a deep learning approach. In IEEE Global Communications Conference (GlobeCom 2015). 1-6.

[44] Xuyu Wang, Lingjun Gao, Shiwen Mao, and Santosh Pandey. 2015. DeepFi: Deep learning for indoor fingerprinting using channel state information. In IEEE Wireless Communications and Networking Conference (WCNC 2015). 1666-1671.

[45] Yi Wang, Xinli Jiang, Rongyu Cao, and Xiyang Wang. 2015. Robust indoor human activity recognition using wireless signals. Sensors 15, 7 (2015), 17195-17208.

[46] Yan Wang, Jian Liu, Yingying Chen, Marco Gruteser, Jie Yang, and Hongbo Liu. 2014. E-eyes: Device-free location-oriented activity identification using fine-grained WiFi signatures. In the 20th annual International Conference on Mobile Computing and Networking (MobiCom 2014). 617-628.

[47] Kaishun Wu, Jiang Xiao, Youwen Yi, Dihu Chen, Xiaonan Luo, and Lionel M Ni. 2013. CSI-based indoor localization. IEEE Transactions on Parallel and Distributed Systems 24, 7 (2013), 1300-1309.

[48] Muchen Wu, Parth H Pathak, and Prasant Mohapatra. 2015. Monitoring building door events using barometer sensor in smartphones. In the 2015 ACM International Joint Conference on Pervasive and Ubiquitous Computing (UbiComp 2015). 319-323.

Proceedings of the ACM on Interactive, Mobile, Wearable and Ubiquitous Technologies, Vol. 1, No. 3, Article 88. Publication date: September 2017. 


\section{$88: 28 \cdot$ K. Ohara et al.}

[49] Chenren Xu, Bernhard Firner, Yanyong Zhang, Richard Howard, Jun Li, and Xiaodong Lin. 2012. Improving RF-based device-free passive localization in cluttered indoor environments through probabilistic classification methods. In International Conference on Information Processing in Sensor Networks (IPSN 2012). 209-220.

[50] Jian Bo Yang, Minh Nhut Nguyen, Phyo Phyo San, Xiao Li Li, and Shonali Krishnaswamy. 2015. Deep convolutional neural networks on multichannel time series for human activity recognition. In the 24th International Joint Conference on Artificial Intelligence (IJCAI 2015). 25-31.

[51] Moustafa Youssef, Matthew Mah, and Ashok Agrawala. 2007. Challenges: Device-free passive localization for wireless environments. In the 13th annual ACM International Conference on Mobile Computing and Networking (MobiCom 2007). 222-229.

[52] Ming Zeng, Le T Nguyen, Bo Yu, Ole J Mengshoel, Jiang Zhu, Pang Wu, and Joy Zhang. 2014. Convolutional neural networks for human activity recognition using mobile sensors. In 6th International Conference on Mobile Computing, Applications and Services (MobiCASE 2014). 197-205.

[53] Daqing Zhang, Hao Wang, Yasha Wang, and Junyi Ma. 2015. Anti-fall: A non-intrusive and real-time fall detector leveraging CSI from commodity WiFi devices. In International Conference on Smart homes and health Telematics (ICOST 2015). 181-193.

[54] Chunting Zhou, Chonglin Sun, Zhiyuan Liu, and Francis Lau. 2015. A C-LSTM neural network for text classification. arXiv preprint arXiv:1511.08630 (2015).

Received February 2017; revised May 2017; accepted July 2017

Proceedings of the ACM on Interactive, Mobile, Wearable and Ubiquitous Technologies, Vol. 1, No. 3, Article 88. Publication date: September 2017. 